Research Article

\title{
Existence Theorem for Impulsive Differential Equations with Measurable Right Side for Handling Delay Problems
}

\author{
Z. Lipcsey, ${ }^{1}$ J. A. Ugboh, ${ }^{1}$ I. M. Esuabana $\left(\mathbb{D},{ }^{1}\right.$ and I. O. Isaac ${ }^{2}$ \\ ${ }^{1}$ Department of Mathematics, University of Calabar, Calabar, Nigeria \\ ${ }^{2}$ Department of Mathematics, Akwa Ibom State University, Mkpat-Enin, Nigeria
}

Correspondence should be addressed to I. M. Esuabana; esuabana@unical.edu.ng

Received 7 August 2019; Revised 12 May 2020; Accepted 18 May 2020; Published 26 June 2020

Academic Editor: Nan-Jing Huang

Copyright (c) 2020 Z. Lipcsey et al. This is an open access article distributed under the Creative Commons Attribution License, which permits unrestricted use, distribution, and reproduction in any medium, provided the original work is properly cited.

Due to noncontinuous solution, impulsive differential equations with delay may have a measurable right side and not a continuous one. In order to support handling impulsive differential equations with delay like in other chapters of differential equations, we formulated and proved existence and uniqueness theorems for impulsive differential equations with measurable right sides following Caratheodory's techniques. The new setup had an impact on the formulation of initial value problems (IVP), the continuation of solutions, and the structure of the system of trajectories. (a) We have two impulsive differential equations to solve with one IVP $\left(\varphi\left(\sigma_{0}\right)=\xi_{0}\right)$ which selects one of the impulsive differential equations by the position of $\sigma_{0}$ in $\left[a, b_{\gamma}\right]$. Solving the selected IVP fully determines the solution on the other scale with a possible delay. (b) The solutions can be continued at each point of $(\alpha, \beta) \times \Omega_{0}=: \Omega$ by the conditions in the existence theorem. (c) These changes alter the flow of solutions into a directed tree. This tree however is an in-tree which offers a modelling tool to study among other interactions of generations.

\section{Introduction}

The innovation of the theory of impulsive systems is manifested in the fact that the time development of the state of such a system forms a mapping of bounded variation instead of continuously differentiable solution of a differential equation [1-8].

The motivation for this research came from two observations, which arose in the theory and applications of impulsive differential equations.

One is the effect of the discontinuity of the right side of the impulsive differential equation, originating from the discontinuity of solutions of delayed equations. A discontinuity of the first order of the solution at a time point may create a set of discontinuity points on the right side (dynamics) of the equation in later time points. This changes the right side to a measurable function of the time instead of a continuous one.

The second issue comes from the representation of a function of bounded variation in terms of two integral forms. In the "usual" representation, the absolute continuous part is a function of time $t$ and the singular part is a function of the singular timer (impulse timer in impulsive differential equations) $\tau$, while in the other representation, the function of $\tau$ is the absolute continuous part and the function of $t$ is singular. In impulsive differential equations, the first form is in use.

The purpose of this paper, therefore, is to analyse and formulate the concept of initial value problem suitable to initialize the obtained pair of impulsive differential equations having measurable right sides and to give conditions for the existence, uniqueness, and continuation of solutions. The existence of solutions of ordinary differential equations with measurable right side has been widely covered by Caratheodory [9]. Therefore, our approach will start from Caratheodory's existence theorem.

The analysis and proof are presented in the following steps.

After giving a brief summary of processes described by impulsive differential equation-Bainovian model and introduction of the system time $t$ and the impulse control time $\tau$, we discussed how existence, uniqueness, and continuation 
are handled in impulsive differential equations with continuous right side.

The analysis of problems arising from delayed equations and their handling leads us to the necessity of the formulation of the extended concept of impulsive differential equations and analysis of the difference between the Bainovian and extended models.

1.1. Systems Described by Impulsive Differential Equations. The Bainovian model for the simplest case is as follows. Let the process evolve in a period of time $T(T=(\alpha, \beta) \subset R$ is an open interval). Let $\Omega_{0} \subset R^{n}$ be an open set and $\Omega:=T \times \Omega_{0}$. Let $f: \Omega \longrightarrow R^{n}$ be an at least continuous mapping, which in addition may fulfill local Lipschitz condition in its variable $x \in R^{n}$, for each fixed $t, \forall(t, x) \in \Omega$. Let $H \subset Z$ be an infinite subset of $Z$ ( $H=N$ or $H=Z$ will be used). Then, let the time sequence $S_{H}=\left\{t_{k}\right\}_{k \in H} \subset T$ be increasing without accumulation points in $T$ and $t_{k} \longrightarrow \alpha, k \longrightarrow-\infty$ and $t_{k} \longrightarrow \beta, k \longrightarrow \infty \quad$ (equivalently, $\quad \forall m, M \in T, m<$ $M,[m, M] \cap S_{H} \subset T$, is a finite set). Let $g: S_{H} \times R^{n} \longrightarrow R^{n}$ be continuous and may fulfill Lipschitz condition in its variable $x, \forall\left(t_{k}, x\right) \in \Omega$. Then, the controlling impulsive differential equation is given by

$$
\begin{cases}x^{\prime}(t)=f(t, x(t)), & \forall t \in T \backslash S_{H}, \\ \Delta x\left(t_{k}\right)=g\left(t_{k}, x\left(t_{k}\right)\right), & \forall t_{k} \in S_{H},\end{cases}
$$

where $(t, x(t)) \in \Omega$.

The impulsive differential equation (1) can be rewritten as an integral equation. We define an ascending step function $\tau: R \longrightarrow Z$ with unit jumps at the impulse points:

$$
\tau(t):=k-1, \quad \text { if } t_{k-1} \leq t<t_{k}, \forall t_{k} \in S_{Z} .
$$

If $H=N$, then $\tau(t)=0 \forall t<t_{1}$.

Corollary 1. The function $\tau: R \longrightarrow R$ is a singular ascending function in $t$ which means as an ascending function it is differentiable almost everywhere and

$$
\frac{\mathrm{d} \tau(t)}{\mathrm{d} t}=0 \quad \text { almost everywhere. }
$$

The singular ascending function $\tau$ defines a singular measure $\tau$ on the Borel sets of $R$. The domain of the function $g$ is extended to the $\widetilde{g}: \Omega \longrightarrow R^{n}$ from the set $S_{H} \times \Omega_{0} \subset \Omega$.

With measures $\tau$ and $\tilde{g}$, equation (1) can be rewritten in an integral form:

$$
\begin{aligned}
& x(t)=x_{0}+\int_{t_{0}}^{t}(f(s, x(s)) \mathrm{d} s+\widetilde{g}(s, x(s)) \mathrm{d} \tau), \quad t_{0}<t \in T, \\
& x\left(t_{0}\right)=x_{0}, \quad t_{0} \in T \backslash S_{H}, x_{0} \in \Omega_{0} .
\end{aligned}
$$

The technical details of these facts are discussed in [10].
Equation (4) has two measures; therefore, it does not look like an integral equation of an (impulsive) differential equation. We will change the parametrization of this equation.

Let $v^{\lambda}(t):=t+\tau^{\lambda}(t)=i d_{\left[a, b_{\lambda}\right]}(t)+\tau^{\lambda}(t)$ ( $\tau^{\lambda}$ represents $\tau$ as defined in Corollary 1$)$, which is a strictly ascending function $v^{\lambda}:\left[a, b_{\lambda}\right] \longrightarrow\left[a, b_{\nu}\right]$ with $b_{\nu}:=b_{\lambda}+\tau^{\lambda}\left(b_{\lambda}\right)-$ $\tau^{\lambda}(a)$. As an ascending function $v^{\lambda}$ has a left- and a rightcontinuous version,

$$
\begin{array}{ll}
\mu_{\lambda,-}^{\lambda}(t):=v^{\lambda}(t-0), & \forall t \in\left[a, b_{\lambda}\right], \\
\mu_{\lambda,+}^{\lambda}(t):=v^{\lambda}(t+0), & \forall t \in\left[a, b_{\lambda}\right] .
\end{array}
$$

The mappings defined in (5) give us an increasing function $\widehat{\mu}_{\lambda}:\left[a, b_{\nu}\right] \longrightarrow\left[a, b_{\lambda}\right]$ defined as follows:

$$
\begin{aligned}
\widehat{\mu}_{\lambda}(t) & :=s, \quad \forall t \in\left[a, b_{\nu}\right], \exists s \in\left[a, b_{\lambda}\right] \text { s.t. } t \in\left[\mu_{\lambda,-}^{\lambda}(s), \mu_{\lambda,+}^{\lambda}(s)\right], \\
\widehat{\mu}_{\lambda} \cdot \mu_{\lambda,-}^{\lambda}(t) & =\widehat{\mu}_{\lambda} \cdot \mu_{\lambda,-}^{\lambda}(t)=i d_{\left[a, b_{\lambda}\right]} .
\end{aligned}
$$

Note that $\widehat{\mu}_{\lambda}$ is one-to-one on the set of continuity points ( $\left[\mu_{\lambda,-}^{\lambda}(s), \mu_{\lambda,+}^{\lambda}(s)\right]$ is singleton if $\left.\mu_{\lambda,-}^{\lambda}(s)=\mu_{\lambda,+}^{\lambda}(s)\right)$. The ascending function $i d_{\left[a, b_{\lambda}\right]}(t)$ is continuous in $\left[a, b_{\lambda}\right]$ while $\tau$ and $v$ may have a countable set of discontinuity points:

$$
D_{\lambda}:=\left\{t \mid \tau^{\lambda}(t-0) \neq \tau^{\lambda}(t+0)\right\}=\left\{t \mid \nu^{\lambda}(t-0) \neq \nu^{\lambda}(t+0)\right\} .
$$

Hence, $\tau^{\lambda}$ and $\nu^{\lambda}$ are continuous in $\left[a, b_{\lambda}\right] \backslash D_{\lambda}$.

1.2. Absolute Continuity and Singularity. Let $v^{\lambda}$ denote the measure defined by the strictly ascending function $v^{\lambda}$. The integral in equation (4) is the sum of integrals with two measures $\lambda^{\lambda}$ and $\tau^{\lambda}$. Both measures $\lambda^{\lambda}$ and $\tau^{\lambda}$ are absolute continuous with respect to $v^{\lambda}$; therefore, both can be written as an integral of the Radon-Nikodym derivatives [11]. With notations $\rho_{\lambda}=\mathrm{d} \lambda^{\lambda} / \mathrm{d} \nu^{\lambda}$ and $\rho_{\tau}=\mathrm{d} \tau^{\lambda} / \mathrm{d} \nu^{\lambda}$, the following important properties are formulated.

Let $\quad N_{\tau}^{\lambda}:=\left\{\left(\mathrm{d} \tau^{\lambda} / \mathrm{d} \nu^{\lambda}\right)>0\right\}$ and $N_{\lambda}^{\lambda}:=\left(N_{\tau}^{\lambda}\right)^{\prime} . \quad$ Then, $\lambda^{\lambda}\left(N_{\tau}^{\lambda}\right)=0=\tau^{\lambda}\left(N_{\lambda}^{\lambda}\right)$ and

$$
\rho_{\lambda}+\rho_{\tau}=\mathrm{d}\left(\lambda^{\lambda}+\tau^{\lambda}\right) / \mathrm{d} \nu^{\lambda}=\mathrm{d} \tau^{\lambda} / \mathrm{d} \nu^{\lambda}+\mathrm{d} \lambda^{\lambda} / \mathrm{d} \nu^{\lambda}=1 .
$$

Moreover, let

$$
\begin{aligned}
& N_{\lambda}^{v}:=\widehat{\mu}_{\lambda}^{-1}\left(N_{\lambda}^{\lambda}\right) \subset\left[a, b_{\nu}\right], \\
& N_{\tau}^{v}:=\widehat{\mu}_{\lambda}^{-1}\left(N_{\tau}^{\lambda}\right) \subset\left[a, b_{\nu}\right], \\
& N_{\lambda}^{v} \cup N_{\tau}^{v}=\left[a, b_{\nu}\right], \\
& N_{\lambda}^{v} \cap N_{\tau}^{v}=\varnothing .
\end{aligned}
$$

These Radon-Nikodym derivatives enable us to rewrite equation (4) using one measure $v^{\lambda}$ as follows: 


$$
\begin{aligned}
& x(t)=x_{0}+\int_{t_{0}}^{t}\left(f(s, x(s)) \frac{\mathrm{d} \lambda^{\lambda}}{\mathrm{d} \nu^{\lambda}}+\tilde{g}(s, x(s)) \frac{\mathrm{d} \tau^{\lambda}}{\mathrm{d} \nu^{\lambda}}\right) \mathrm{d} \nu^{\lambda}, \quad t \in T, \\
& x\left(t_{0}\right)=x_{0}, \\
& t_{0} \in T \backslash S_{H}, x_{0} \in \Omega_{0} . \\
& D_{\lambda}^{v}:=\underset{t \in D_{\curlywedge}}{\cup}\left[\mu_{\lambda,-}(t), \mu_{\lambda,+}(t)\right) \subset\left[a, b_{\gamma}\right] .
\end{aligned}
$$

The details of these assertions are in paper [10].

1.3. Measures. The mappings $\tau^{\lambda}$ and $\nu^{\lambda}$ are ascending not continuous functions, with a common set of discontinuity points $D_{\lambda}$. Therefore, the measures $\tau^{\lambda}$ and $\nu^{\lambda}$ are defined on the semiring $P_{\nu,\left[a, b_{1}\right], c}=\left\{[s, t) \mid s, t \in\left[a, b_{\lambda}\right] \backslash D_{\lambda}, s \leq t\right\}$ and the measures $\tau^{\lambda,}([s, t)):=\tau^{\lambda}(t)-\tau^{\lambda}(s)$ and $\nu^{\lambda}([s, t)):=v^{\lambda}(t)-$ $\nu^{\lambda}(s), \forall[s, t) \in P_{\nu,\left[a, b_{\lambda}\right], c}$ can be extended to $\mathscr{B}_{\gamma,\left[a, b_{\lambda}\right], c}=$ $\sigma\left(\mathscr{P}_{\nu,\left[a, b_{\lambda}\right], c}\right)$.

The mappings $\mu_{\lambda,-}^{\lambda}, \mu_{\lambda,+}^{\lambda}:\left[a, b_{\lambda}\right] \longrightarrow\left[a, b_{v}\right]$ map the set of discontinuity points $D_{\lambda}$ into the set of left-closed rightopen intervals:

$$
\mathscr{D}_{\lambda}^{\nu}:=\left\{\left[\mu_{\lambda,-}(t), \mu_{\lambda,+}(t)\right) \mid t \in D_{\lambda}\right\},
$$

and the set of discontinuity points in $\left[a, b_{\gamma}\right]$ is
Moreover, the mappings $\mu_{\lambda,-}^{\lambda}$ and $\mu_{\lambda,+}^{\lambda}$ are bijective on the set of continuity points $\left[a, b_{\gamma}\right] \backslash D_{\lambda}^{\nu}$ and $\mu_{\lambda,-}(t)=$ $\mu_{\lambda,+}(t), \forall t \in\left[a, b_{\lambda}\right] \backslash D_{\lambda}$.

The mappings $\mu_{\lambda_{-}}^{\lambda}, \mu_{\lambda_{+}+}^{\lambda}:\left[a, b_{\lambda}\right] \longrightarrow\left[a, b_{\nu}\right]$ transform $[s, t) \in P_{\nu,\left[a, b_{1}\right], c} \longrightarrow\left[\nu^{\lambda}(s), \nu^{\lambda}(t)\right)=\left[\mu_{\lambda,-}^{\lambda}(s), \mu_{\lambda,-}^{\lambda}(t)\right)=$ $\left[\mu_{\lambda,+}^{\lambda}(s), \mu_{\lambda,+}^{\lambda}(t)\right) \in P_{\nu,\left[a, b_{\nu}\right], c}^{v} \quad$ by $\quad P_{\nu,\left[a, b_{\nu}\right], c}^{v}:=\{[u, v) \mid u, v \in$ $\left.\left[a, b_{\gamma}\right] \backslash D_{\lambda}^{\nu}\right\}$.

The measure $\nu_{\lambda}^{v}$ on $P_{\nu,\left[a, b_{v}\right], c}^{v}$ is defined by $v_{\lambda}^{\nu}([u, v)):=$ $v-u=\mu_{\lambda,-}\left(\widehat{\mu}_{\lambda}^{\lambda}(v)\right)-\mu_{\lambda,-}\left(\widehat{\mu}_{\lambda}^{\lambda}(u)\right)=\nu_{\lambda}\left(\widehat{\mu}_{\lambda}^{\lambda}([u, v))\right) \forall[u, v) \in$ $P_{\nu,\left[a, b_{v}\right], c}^{v}$. Also, if $[s, t) \in P_{\nu,\left[a, b_{\lambda}\right], c}$ then $\left[\mu_{\lambda,-}(s), \mu_{\lambda,-}(t)\right) \in$ $P_{\nu,\left[a, b_{\gamma}\right], c}^{v} \quad$ and $\quad \nu_{\lambda}^{\nu}\left(\left[\mu_{\lambda,-}(s), \mu_{\lambda,-}(t)\right)\right)=\mu_{\lambda,-}(t)-\mu_{\lambda,-}(s)=$ $\nu_{\lambda}([s, t))$. Let the smallest $\sigma$-algebra containing the semiring $P_{\lambda,\left[a, b_{v}\right], c}^{v}$ be $\mathscr{B}_{\lambda,\left[a, b_{v}\right], c}^{v}:=\sigma\left(P_{\lambda,\left[a, b_{\gamma}\right], c}^{v}\right)$ with the extended measure $v_{\lambda}^{v}$ on it. Then, the following relations hold:

$$
\begin{aligned}
& \int_{a}^{\mu_{\lambda,+}(t)} f \cdot \widehat{\mu}_{\lambda} \mathrm{d} \nu_{\lambda}^{\nu}=\int_{a}^{t} f \mathrm{~d} \nu^{\lambda}, \quad \forall[a, t) \in \mathscr{B}_{\nu,\left[a, b_{\lambda}\right], c}, \forall f \in L_{1}\left(\nu^{\lambda}, \mathscr{B}_{\nu,\left[a, b_{\lambda}\right], c}\right), \\
& \int_{a}^{\sigma} f \mathrm{~d} \nu_{\lambda}^{\nu}=\int_{a}^{\widehat{\mu}_{\lambda}(\sigma)} f \cdot \mu_{\lambda,-} \mathrm{d} \nu^{\lambda}, \quad \forall[a, \sigma) \in \mathscr{B}_{\lambda,\left[a, b_{\nu}\right], c}^{v}, \forall f \in L_{1}\left(\nu_{\lambda}^{\nu}, \mathscr{B}_{\lambda,\left[a, b_{\nu}\right], c}^{v}\right) .
\end{aligned}
$$

The details of these assertions are in paper [10].

1.4. Existence and Continuation of a Solution. Existence, uniqueness, and continuation of solutions are fundamental issues for differential equations of all kinds. These issues, therefore, have been studied by many authors $[1,2,12-19]$ just to mention a few. These articles consider initial value problems and boundary value problems for impulsive differential equations with an at least continuous right side $f$ in $\Omega$ or in addition to the continuity $f$ fulfills Lipschitz condition in its spatial variables; therefore, the analysis is based on Cauchy-Peano's or Piccard-Lindelöf's existence theorems [9]. The sources of discontinuities are arranged so that any closed bounded interval contains finite number of discontinuity points of the first type. We give a summary of these systems by pointing out the major differences in properties compared with the ordinary differential equations. If the initial value problem is not prescribed at a discontinuity time point then Cauchy-Peano's or Piccard-Lindelöf's existence theorems [9] provide solutions extendible in line with the rules of ordinary differential equations.

Continuation: by the assumption that the function $f: \Omega \longrightarrow R^{n}$ fulfills the conditions of an existence theorem (Cauchy-Peano's or Piccard-Lindelöf's existence theorem [9]), in $\Omega$, any solution that reaches a point $(s, \varphi(s)) \in$ $\Omega, t_{0}<s$ has a continuation on an interval $[s, s+\delta)$ with a suitable $\delta>0$. The process stops only at a boundary point $(s, \varphi(s)) \in \partial \Omega$. The impulses, however, change this scenario. If the solution reaches $\left(t_{j}, \varphi\left(t_{j}-0\right) \in \Omega, t_{j} \in S_{H}\right.$, then there is a continuation to $\varphi\left(t_{j}+0\right)=\varphi\left(t_{j}-0\right)+g\left(t_{j}, \varphi\left(t_{j}-0\right)\right)$. If $\left(t_{j}, \varphi\left(t_{j}+0\right)\right) \notin \Omega$, the process stops at $\left(t_{j}, \varphi\left(t_{j}-0\right)\right)=\left(t_{j}, \varphi\left(t_{j}\right)\right) \in \Omega$. Otherwise, if $\left(t_{j}, \varphi\left(t_{j}+\right.\right.$ $0))) \in \Omega$, then it has a continuation as described above. The jump mapping $\mathscr{J}\left(t_{j}, y\right):=\left(t_{j}, y+g\left(t_{j}, y\right)\right)$ having a value $\forall y \in \Omega_{0}$ gives further changes. All solutions reaching the impulse point $t_{j}$ continue to $\varphi\left(t_{j}+0\right)$. If $\mathscr{F}: S_{H} \times$ $\Omega_{0} \longrightarrow S_{H} \times \Omega_{0} \subset \Omega$, then each trajectory is continued beyond the impulse time point. However, the range $\mathscr{R}(\mathscr{J})$ may be a proper subset of $S_{H} \times \Omega_{0}$. Then, the solution $\varphi$ of equation (1) fulfilling the initial value problem $\left(s_{0}, \varphi\left(s_{0}\right)\right) \in\left(S_{H} \times\left(\Omega_{0}\right) \backslash \mathscr{R}(\mathscr{J})\right)$ has no history, will have no continuation on the interval $\left(s_{0}-\delta, s_{0}\right]$, and the continuation will exist on $\left[s_{0}, s_{0}+\delta\right)$ only. These properties are also mentioned in $[1,14]$.

1.4.1. Impulsive Delayed Differential Equations. The research on impulsive delay differential equations is very intensive as the cited list of some of the publications $[2,20-37]$. The right side of the equations is still continuous or may fulfill Lipschitz conditions and sustains the finiteness of discontinuity points in closed bounded time intervals. The model of delayed impulsive systems developed by Bainov and his group [38] is based on a discrete set $S_{H} \subset R$ of impulse points with no accumulation points in any bounded 
interval. In these models, the delayed impact uses these same set $S_{H}$ of impulse points which regulate the occurrence of impulses at impulse time points which maybe a costly assumption. In some other approaches, different ways are used to meet the condition of local finiteness of the set of discontinuity points. Hence, to guarantee the local finiteness of discontinuity points of the right side is increasingly difficult in delayed systems, it is worth to see the effect of delay on the right side as presented in the following examples.

Important examples: let the right side of the impulsive differential equation be defined as follows. Let $[a, b] \supset(\alpha, \beta), \eta:=(\alpha+\beta) / 2$ and let $\alpha-h=: \gamma \in(a, b], h>0$. Then, let

$$
\begin{array}{ll}
f(x(t-\vartheta(t)), x(t)):=x(t)+x(t-\vartheta(t)), & \forall t \in(\alpha, \beta), \\
g((x(t-\vartheta(t)), x(t)):=2 x(t)+x(t-\vartheta(t)), & \forall t \in(\alpha, \beta), \\
S_{H} \cap(\alpha, \beta)=\varnothing, & \gamma \in S_{H} .
\end{array}
$$

Let the right continuous solution of the initial value problem of the equation be

$$
\begin{array}{ll}
x(t)=x_{0}+\int_{t_{0}}^{t}(f(x(s-\vartheta(s)), x(s)) \mathrm{d} s+g(x(s-\vartheta(s)), x(s)) \mathrm{d} \tau), & t \in\left[t_{0}, b\right], \\
x\left(t_{0}\right)=x_{0}, & t_{0} \in(a, \gamma) \backslash S_{H} .
\end{array}
$$

Assume that $x(\gamma-0)=1$ and $x(\gamma+0)=-1$.

Let $\vartheta$ be continuous ascending function, $\vartheta(t)<t, \forall t \in$ $(\alpha, \beta)$. Let $u(t):=t-\vartheta(t) \Rightarrow \vartheta(t)=t-u(t)$.

We will now show some simple examples to demonstrate that delay equations may lead to differential equations with measurable right sides:

(1) Let $u(t):=\gamma-\varepsilon(t-\alpha)(\eta-t)(\beta-t) \forall t \in(\alpha, \beta)$. This will give $u(\alpha)=u(\eta)=u(\beta)=\gamma$; hence, $x(\eta-0)=$
$1 \neq-1=x(\eta+0)$. Therefore, $f(x(t-\vartheta(t)), x(t))$ has both left and right limits which are not the same. Hence, $f$ is measurable and not a continuous function of $t$ in $[a, b]$ and $\vartheta$ is ascending with suitable selection of $\varepsilon$.

(2) Let $t_{j}:=\eta-((\beta-\alpha) / 2 j), 1 \leq j<\infty, t_{j} \nearrow \eta$ if $j \longrightarrow$ $\infty$. Then,

$$
u(t):= \begin{cases}\gamma+\frac{(-1)^{j}}{2^{2}(\eta-\alpha)}\left(t-t_{j}\right)^{2}\left(t_{j+1}-t\right)^{2}, & \text { if } t_{j} \leq t \leq t_{j+1}, j \in N . \\ 0, & \text { otherwise. }\end{cases}
$$

The function $u(t)=\gamma, \forall t=t_{i}$, and $u(t)<\gamma$ if $t_{i}<t<t_{i+1}$ and $i$ is odd and $u(t)>\gamma$ if $t_{i}<t<t_{i+1}$ and $i$ is even. Hence, $x\left(t_{i}-0\right)=-1$ and $x\left(t_{i}+0\right)=1$ if $i$ is odd and $x\left(t_{i}-0\right)=1$ and $x\left(t_{i}+0\right)=-1$ if $i$ is even. Hence, $\lim \sup _{t>\eta} x(t-\vartheta(t))=1$ and $\lim \inf _{t>\eta} x(t-$ $\vartheta(t))=-1$.

The delayed solution $x(t-\vartheta(t))$ with this delay has no left limit hence no limit at $\eta \in(\alpha, \beta)$. Furthermore, there is no limit at $t_{j}, \forall 1 \leq j<\infty$. Hence, $f$ with delayed arguments is measurable and not continuous function of $t$ in $[a, b]$.

(3) Continuous descending delay leads to bijective mapping of the impulse points; hence, in this case, there are no accumulation points of the images of impact points but the statement about measurable right side remains valid.

Conclusion 1. Examples 1 and 3 can be handled with the help of the existence theorems such as Cauchy-Peano's or Piccard-Lindelöfs [9] since the discontinuity points have no accumulation points. The second example, however, requires limit theorems and additional reasoning. If Example 2 is combined with the construction of Cantor's triadic set [39], then we get a set of discontinuity points of continuum cardinality. This means that alternative approach may be necessary to handle such initial value problems.

\section{Extended Impulsive Differential Equations and Existence of Their Solution}

The extended impulsive differential equations mean changing some basic assumptions used in Bainov's model as described in equation (1) or in rewritten form in equations (3) and (10). Major changes include the time control of the impulses may have infinite discontinuities but has to be of bounded variation on every closed bounded interval, and the system dynamics is measurable as a function of the time and not necessarily continuous.

2.1. The Extended Impulsive Differential Equations. Let the process evolve in an open time interval $T \subset R$ and in an open set $T \times \Omega_{0}=: \Omega \subset T \times R^{n}$. Let $f, g: \Omega \longrightarrow R^{n}$ be measurable functions in the time variable $t$ for each fixed spatial value $x \in \Omega_{0}$, and continuous/Lipschitz-continuous functions in the spatial variable $x$ for each fixed $t \in T$. 
Let $\tau: T \longrightarrow R^{+}$be a singular ascending function of the time parameter $t$ as the singular "impulse timer". It is important to see that $\tau$ may have a countably infinite set of jump points, where the total lengths of these jumps must be bounded on any closed bounded interval. Using equation (8), we can rewrite the Radon-Nikodym derivatives in terms of characteristic functions of the sets $N_{\lambda}^{\lambda}$ and $N_{\lambda}^{\tau}$ as follows:

$$
\frac{\mathrm{d}\left(\lambda^{\lambda}+\tau^{\lambda}\right)}{\mathrm{d} v^{\lambda}}=\frac{\mathrm{d} \lambda^{\lambda}}{\mathrm{d} \nu^{\lambda}}+\frac{\mathrm{d} \tau^{\lambda}}{\mathrm{d} \nu^{\lambda}}=1 \Rightarrow \frac{\mathrm{d} \lambda^{\lambda}}{\mathrm{d} \nu^{\lambda}}=\chi_{N_{\lambda}^{\lambda}} \text { and } \frac{\mathrm{d} \tau^{\lambda}}{\mathrm{d} \nu^{\lambda}}=\chi_{N_{\lambda}^{\tau}} .
$$

Putting these into equation (10) and changing $\widetilde{g}$ with $g$, we get the extended impulsive differential equation in $t$-scale as

$$
\begin{array}{ll}
x(t)=x_{0}+\int_{t_{0}}^{t}\left(f(s, x(s)) \chi_{N_{\lambda}^{\lambda}}+g(s, x(s)) \chi_{N_{\tau}^{\lambda}}\right) \mathrm{d} v^{\lambda}, & t \in T, \\
x\left(t_{0}\right)=x_{0} & t_{0} \in T \backslash S_{H}, x_{0} \in \Omega_{0} .
\end{array}
$$

The integral transformations discussed in Section 2.3.5 will give a similar result in both $\nu$-scale and $\tau$-scale. We will handle the $\nu$-scale representation first. Let $f^{v}:=$ $\left(\left(f(s, x(s)) \chi_{N_{\lambda}^{\lambda}}\right) \cdot \widehat{\mu}_{\lambda}^{\nu}: N_{\lambda}^{v} \longrightarrow R^{n} \quad\right.$ and $\quad g^{v}:=((g(s, x(s))$ $\left.\chi_{N_{\tau}^{\lambda}}\right) \cdot \widehat{\mu}_{\lambda}^{\nu}: N_{\tau}^{v} \stackrel{\lambda}{\longrightarrow} R^{n}$. Then, let

$$
h^{v}:=f^{v} \chi_{N_{\lambda}^{v}}+g^{v} \chi_{N_{\tau}^{v}}:\left[a, b_{\nu}\right] \times \Omega_{0} \longrightarrow R^{n}
$$

be the measurable right side of the extended impulsive differential equation in $v$-scale.

We will use the notations $\left[a, b_{\lambda}\right] \subset T$ for $t$-scale, $\left[a, b_{\gamma}\right]$ for the generated $\nu$-scale, and $\left[a, b_{\tau}\right]$ for $\tau$-scale to get the advantages of compact sets.

In Section 2.2, we will discuss the main results of this paper which is formulation of the extension of Caratheodory's existence theorem for the extended impulsive differential equations with measurable right side. The basis of our discussion is the approach presented in pg. 43 in [9].

2.2. Caratheodory's Theorem. We present Caratheodory's existence theorem in $R^{n}$ as it is presented in the cited pages 42-43 for one dimension.

We are considering a process on an open set $\mathcal{S} \subset \Omega \subset R \times R^{n}$. Let $f: S \longrightarrow R^{n}$ be a function not necessarily continuous.

Problem (E): find an interval $I \subset[a, b]$ and an absolute continuous function $\varphi: I \subset(a, b) \longrightarrow R^{n}$ such that

$$
\begin{array}{r}
(t, \varphi(t)) \in S, \\
\varphi^{\prime}(t)=f(t, \varphi(t)), \quad \text { almost all } t \in I .
\end{array}
$$

Then, the function $\varphi: I \longrightarrow R^{n}$ is a solution of equation (20) in the extended sense.

Caratheodory's existence theorem [9] targets finding a solutions to problem (E) with an initial value $\left(t_{0}, \xi\right) \in \Omega, \varphi\left(t_{0}\right)=\xi$, where the right side is a measurable function of $t$ for each fixed $x \in \Omega_{0}$ on $T \times \Omega_{0}$, where $\varnothing \neq(\alpha, \beta)=T \subset R$ and $\Omega_{0} \subset R^{n}$ are open sets. Caratheodory's condition for the existence of the solution is the existence of a local positive integrable dominant $m:\left(t_{0}-\right.$ $\left.\gamma, t_{0}+\gamma\right) \subset T \longrightarrow R^{+} \backslash\{0\}$ and $\varepsilon>0$ such that $\|f(t, x)\| \leq$ $m(t), \forall(t, x) \in\left(t_{0}-\gamma, t_{0}+\gamma\right) \times B_{\varepsilon}(\xi)$. This condition guarantees that, for any measurable curve $\varphi$ : $\left(t_{0}-\gamma\right.$, $\left.t_{0}+\gamma\right) \longrightarrow B_{\varepsilon}(\xi)$, the measurable function $f(t, \varphi(t))$, $t \in\left(t_{0}-\gamma, t_{0}+\gamma\right)$ is integrable in the intervals $\left(t_{0}-\gamma\right.$, $\left.t_{0}\right)$ and $\left(t_{0}, t_{0}+\gamma\right)$ by $\|f(t, \varphi(t))\| \leq m(t), \forall t \in\left(t_{0}-\gamma\right.$, $\left.t_{0}+\gamma\right)$.

Definition 1. Let a point $\left(t_{0}, \xi\right) \in \Omega$ be selected and let $\mathscr{R}_{\delta, \varepsilon}\left(t_{0}, \xi\right):=\left(t_{0}-\delta, t_{0}+\delta\right) \times B_{\varepsilon}(\xi) \subset \Omega, 0<\delta, \varepsilon$ be a cylinder. Let $f: \Omega \longrightarrow R^{n}$ be a measurable function. Then, we will call $f$ locally $t$-integrable at a point $\left(t_{0}, \xi\right) \in \Omega$ if there exists a cylinder $\mathscr{R}_{\delta, \varepsilon}\left(t_{0}, \xi\right) \subset \Omega, 0<\delta, \varepsilon$ and an dominating integrable function (D. I. F.) $m:\left(t_{0}-\delta, t_{0}+\delta\right) \longrightarrow R^{+} \backslash\{0\}$ to $f$ on the cylinder $\mathscr{R}_{\delta, \varepsilon}\left(t_{0}, \xi\right)$ such that $\|f(t, x)\| \leq$ $m(t) \forall(t, x) \in \mathscr{R}_{\delta, \varepsilon}\left(t_{0}, \xi\right)$.

Theorem 1. (Caratheodory). Let $f: \mathcal{S} \longrightarrow R^{n}$ be measurable in $t$ for each fixed $x$, and let it be continuous in $x$ for each fixed $t, \forall(t, x) \in \mathcal{S}$. Let $\left(t_{0}, \xi\right) \in \mathcal{S}$ be a fixed point and let a cylinder $\mathscr{R}_{\delta, \varepsilon}\left(t_{0}, \xi\right) \subset \mathcal{S}$ exist with a dominating integrable function (D.I.F.) $m:\left(t_{0}-\delta, t_{0}+\delta\right) \longrightarrow R^{+} \backslash\{0\}$ to $f$ on the cylinder $\mathscr{R}_{\delta, \varepsilon}\left(t_{0}, \xi\right)$. Then, there exists a solution $\varphi$ of problem (E) in an extended sense in an interval $\left(t_{0}-\beta, t_{0}+\beta\right)$, $0<\beta \leq \delta$, such that $(t, \varphi(t)) \in \mathscr{R}_{\delta, \varepsilon}\left(t_{0}, \xi\right), \forall t \in\left(t_{0}-\beta, t_{0}+\right.$ $\beta)$ and $\varphi\left(t_{0}\right)=\xi$.

Caratheodory actually proved the existence on an interval $\left[t_{0}, t_{0}+\beta\right)$ interval and used this result to prove the existence on $\left(t_{0}-\beta, t_{0}\right]$ by using suitable transformations of symmetry.

Using Caratheodory's theorem we can prove the existence of solution of the extended impulsive differential equation equation on $v$-scale with right side (19), precisely.

Corollary 2. Let $f^{v}: N_{\lambda}^{v} \times \Omega_{0} \longrightarrow R^{n}$ and $g^{\nu}: N_{\tau}^{v} \times$ $\Omega_{0} \longrightarrow R^{n}$; hence, let $h^{v}:=f^{v} \chi_{N^{v}}+g^{v} \chi_{N_{\tau}^{v}}$ be measurable in $\sigma$ for each fixed $x$, and let it be continuous in $x$ for each fixed $\sigma$, $\forall(\sigma, x) \in \Omega$. Let $\left(\sigma_{0}, \xi_{0}\right) \in \Omega$ be a fixed point and let a cylinder $\mathscr{R}_{\delta, \varepsilon}\left(\sigma_{0}, \quad \xi_{0}\right) \subset \Omega$ exist with a D.I.F. $m:\left[\sigma_{0}, \sigma_{0}+\delta\right) \longrightarrow R^{+} \backslash\{0\}$ on $\mathscr{R}_{\delta, \varepsilon}\left(\sigma_{0}, \xi_{0}\right)$ to $h^{\nu}$. Then, there exists an interval $\left[\sigma_{0}, \sigma_{0}+\beta\right), 0<\beta \leq \delta$ for the equation 


$$
\varphi(\sigma)=\xi_{0}+\int_{\sigma_{0}}^{\sigma}\left(f^{v}(v, \varphi(v)) \chi_{N_{\lambda}^{v}}+g^{v}(v, \varphi(v)) \chi_{N_{\tau}^{v}}\right) \mathrm{d} v=\xi_{0}+\int_{\sigma_{0}}^{\sigma} h^{v}(v, \varphi(v)) \mathrm{d} \nu
$$

such that equation (21) has a solution $\varphi$ in that interval such that $(\sigma, \varphi(\sigma)) \in \mathscr{R}_{\delta, \varepsilon}\left(\sigma_{0}, \xi\right), \forall \sigma \in\left[\sigma_{0}, \sigma_{0}+\beta\right)$ and $\varphi\left(\sigma_{0}\right)=$ $\xi_{0}$.

Corollary 3. Let $h^{v}$ defined by equation (15) be $\mathscr{B}_{\lambda,\left[a, b_{\nu}\right], c}^{v}$-measurable as a function of $\sigma$ for each fixed $x$, $\forall(\sigma, x) \in\left[a, b_{\nu}\right] \times \Omega_{0}$ in addition to the conditions of Corollary 2. Then, the solution of initial value problem $\varphi\left(\sigma_{0}\right)=$ $\xi_{0},\left(\sigma_{0}, \xi_{0}\right) \in N_{\lambda}^{v} \times \Omega_{0}$ for equation (17) exists on an interval $\left[\sigma_{0}, \sigma_{0}+\beta\right)$ for a suitable $\beta>0$ and $x_{\lambda}:=\varphi\left(\mu_{\lambda,+}\right)$ is a solution of the initial value problem $x_{\lambda}\left(t_{0}\right)=\xi_{0}$ with $t_{0}=\widehat{\mu}_{\lambda}\left(\sigma_{0}\right)$ imposed on the equation:

$$
\begin{gathered}
\varphi\left(\mu_{\lambda,+}(t)\right)=\xi_{0}+\int_{\sigma_{0}}^{\mu_{\lambda,+}(t)} h^{v}(v, \varphi(v)) \mathrm{d} \nu_{\lambda}^{v}=\xi_{0}+\int_{t_{0}}^{t} h^{\nu}\left(\mu_{\lambda,+}(s), \varphi\left(\mu_{\lambda,+}(s)\right)\right) \mathrm{d} \nu^{\lambda} \Rightarrow \\
x_{\lambda}(t)=\xi_{0}+\int_{t_{0}}^{t} h^{\lambda}\left(\widehat{\mu}_{\lambda}\left(\mu_{\lambda,+}(s), x_{\lambda}(s)\right) \mathrm{d} \nu^{\lambda}=\xi_{0}+\int_{t_{0}}^{t} h^{\lambda}\left(s, x_{\lambda}(s)\right) \mathrm{d} \nu^{\lambda} .\right.
\end{gathered}
$$

This identity follows from equation (13). Details will be discussed later.

Remark 1. Note that the condition $t_{0} \notin D_{\lambda}$ used in Corollary 3 is in the Bainovian initial value problem (4). Therefore, the Bainovian case with measurable right side is covered by this simple example.

Note also that $h^{v}$ can be $\mathscr{B}\left(\left[a, b_{\gamma}\right], v\right)$-measurable which is not covered in this corollary.

Remark 2. With Corollary 2, the discussion about general existence theorem has been finished. The rest of this paper will target to include the solutions of equations with $\mathscr{B}\left(\left[a, b_{\nu}\right], \nu\right)$-measurable right sides and the formulation of the conditions for the existence of solutions in terms of the measurable functions $f^{\lambda}$ and $g^{\tau}$. Some issues will have to be clarified about the initial value problems.

As shown in the example, solutions of the impulsive differential equations are obtained from the absolute continuous solutions on the $v$-scale with the help of suitable transformations. We will develop some extensions of the mappings $\mu_{\lambda,-}^{\lambda}, \mu_{\lambda,+}^{\lambda}$, and $\widehat{\mu}_{\lambda}$.

Uniqueness: the solution of an initial value problem is unique if the right side of the differential equation fulfills local Lipschitz condition [9]. Although there are other conditions for uniqueness, we will demonstrate our presentation on this condition. We concluded in Section 1.4 about continuation of solutions that, for impulsive differential equations, the solution of an initial value problem $\left(t_{0}, \varphi\left(t_{0}\right)\right)=\left(t_{0}, \xi_{0}\right) \in \Omega$ exists on an interval $\left[t_{0}, t_{0}+\delta\right) \subset T$ if the conditions of one of the existence theorems hold $\forall\left(t_{0}, \xi_{0}\right) \in \Omega$.

Let us consider the impact of this condition on an example (originating from [9]). Let the differential equation be as follows:

$$
y^{\prime}= \begin{cases}0, & -\infty<t<-1, \\ \frac{2 y}{t}, & -1 \leq t<0, y \in R, \\ 0, & 0 \leq t<\infty .\end{cases}
$$

The differential equation fulfills local Lipschitz condition $\forall(t, y) \in R^{2}$ on an interval $\left[t, t+\delta_{t}\right), \delta_{t}>0$.

Let $I:=(-\infty,-1) \cup[0, \infty)$; then, for any $\left(t_{0}, y_{0}\right) \in I \times$ $R, L=1$ is a suitable choice as Lipschitz constant in $\left[t_{0}, t_{0}+\delta_{t_{0}}\right) \times B_{\delta_{t_{0}}}\left(y_{0}\right) \subset\left[t_{0}, t_{0}+\delta_{t_{0}}\right] \times \overline{B_{\delta_{t_{0}}}\left(y_{0}\right)} \subset I \times R$, with a suitable $\delta_{t_{0}}>0$.

If $\left(t_{0}, y_{0}\right) \in[-1,0) \times R$ and $\left[t_{0}, t_{0}+\delta_{t_{0}}\right) \subset\left[t_{0}, t_{0}+\right.$ $\left.\delta_{t_{0}}\right] \subset[-1,0)$, then $L=\max \left\{2 /|t| \mid(t, y) \in\left[t_{0}, t_{0}+\delta_{t_{0}}\right] \times\right.$ $\left.\overline{B_{\delta_{t_{0}}}\left(y_{0}\right)}\right\}$ will serve as the Lipschitz constant. The right side fulfills a local Lipschitz condition in a suitable neighbourhood $\left[t_{0}, t_{0}+\delta_{t_{0}}\right) \times B_{\delta_{t_{0}}}\left(y_{0}\right)$ at any $\left(t_{0}, y_{0}\right) \in R^{2}$. Hence, no solution trajectory split into two or more trajectories at any point $t>t_{0}$.

However, the right side does not fulfill Lipschitz condition in any interval $(a, 0], a<0$. The solutions of all initial value problems $y(-1)=y_{1} \in R$ will pass through $\varphi\left(0 ;-1, y_{1}\right)=0$ by the formula $\varphi\left(t ;-1, y_{1}\right)=\left(y_{1} /(-1)^{2}\right) t^{2}$. Therefore, merging of solution trajectories can occur while splitting of trajectories is excluded by having local Lipschitz condition at each point $(t, y) \in R^{2}$ in an interval $[t, t+$ $\left.\delta_{t}\right) \times B_{\delta_{t}}(y)$. However, note that if $\varphi_{1}\left(t_{1}\right)=y_{1}$ and $\varphi_{2}\left(t_{2}\right)=$ $y_{2} \in R,\left(t_{1}, y_{1}\right) \neq\left(t_{2}, y_{2}\right)$ then the two global solutions are different by the initial value problems even if $\varphi_{1}(s)=\varphi_{2}(s)$ holds at an $s>\max \left\{t_{1}, t_{2}\right\} \in R \Rightarrow \varphi_{1}(t)=\varphi_{2}(t), \forall t \geq s$. Hence, the global solutions of two different initial value problems are two different trajectories (not necessarily disjoint trajectories). 
2.3. Timescales and Their Density Functions. This section summarises the concepts which will serve as the basis of most of our coming discussion and were developed in [10] and partly in [40]. We use the notations and concepts formulated in Section 1.1 and our starting point will be the integral equation (4).

We showed that, given $\left[a, b_{\lambda}\right], N_{\lambda}^{\lambda}, N_{\tau}^{\lambda}, \lambda^{\lambda}$, and $\tau^{\lambda}$, we obtain $\left[a, b_{\gamma}\right], N_{\lambda}^{\nu}, N_{\tau}^{v}$, and $\nu_{\lambda}^{\nu}$. We now show the reverse order.
2.3.1. The $t$-Scale from $\nu$-Scale. We show now that $\left[a, b_{\nu}\right], N_{\lambda}^{\nu}, N_{\tau}^{v}$, and $\nu$ determines $\left[a, b_{\lambda}\right], N_{\lambda}^{\lambda}, N_{\tau}^{\lambda}, \lambda^{\lambda}$, and $\tau^{\lambda}$. Since $N_{\lambda}^{\nu}$ and $N_{\tau}^{\nu}$ are measurable sets and $\left[a, b_{\nu}\right]$ is bounded, the characteristic functions of $N_{\lambda}^{\nu}$ and $N_{\tau}^{\nu}$ are $\nu$-integrable. Let the time scale interval be $\left[a, b_{\lambda}\right]$ with $b_{\lambda}^{\tau}:=a+\int_{a}^{b_{\nu}} \chi_{N_{\lambda}^{\nu}} \mathrm{d} \nu$,

$$
\begin{aligned}
\widehat{\mu}_{\lambda}(s) & :=a+\int_{a}^{s} \chi_{N_{\lambda}^{\nu}} \mathrm{d} v \in\left[a, b_{\lambda}\right], & \forall s \in\left[a, b_{\nu}\right], \\
\mu_{\lambda,-}^{\lambda}(s) & :=\inf \widehat{\mu}_{\lambda}^{-1}(\{s\}) \in\left[a, b_{\nu}\right], & \forall s \in\left[a, b_{\lambda}\right], \\
\mu_{\lambda}^{\lambda}(s) & :=\mu_{\lambda,+}^{\lambda}(s):=\sup \widehat{\mu}_{\lambda}^{-1}(\{s\}) \in\left[a, b_{\nu}\right], & \forall s \in\left[a, b_{\lambda}\right], \\
\widehat{\mu}_{\lambda} \cdot \mu_{\lambda,-}^{\lambda}(t) & =\widehat{\mu}_{\lambda} \cdot \mu_{\lambda,-}^{\lambda}(t)=i d\left[a, b_{\lambda}\right], & \\
\nu^{\lambda}(s) & =a+\int_{a}^{\mu_{\lambda,+}^{\lambda}(s)} 1 \mathrm{~d} \nu^{\nu}=a+\int_{a}^{\mu_{\lambda,+}^{\lambda}(s)}\left(\chi_{N_{\lambda}^{v}}+\chi_{N_{\tau}^{v}}\right) \mathrm{d} \nu^{\nu}=a+\lambda^{\lambda}([a, s))+\tau^{\lambda}([a, s)), & \forall s \in\left[a, b_{\lambda}\right],
\end{aligned}
$$

where $v^{\lambda}$ is right continuous. Since $v^{\lambda}$ is strictly ascending, $N_{\lambda}^{\lambda}=\left(\nu^{\lambda}\right)^{-1}\left(N_{\lambda}^{\nu}\right)$ and $N_{\tau}^{\lambda}=\left(\nu^{\lambda}\right)^{-1}\left(N_{\tau}^{\nu}\right)$ and $\tau^{\lambda}$ is singular with respect to $\lambda^{\lambda}$ and $\lambda^{\lambda}$ is singular with respect to $\tau^{\lambda}$ by

$$
\lambda^{\lambda}\left(N_{\tau}^{\lambda}\right)=0=\tau^{\lambda}\left(N_{\lambda}^{\lambda}\right)
$$

$S_{H}:=D_{\lambda}:=\left\{t \mid \mu_{\lambda,-}(t) \neq \mu_{\lambda,+}(t), t \in\left[a, b_{\lambda}\right]\right\} \quad$ is the countable set of discontinuity points in $t$-scale. From now on, we will use $D_{\lambda}$ in the place of $S_{H}$.

Hence, based on equation (22) with $h^{\nu}, x_{\lambda}:=\varphi\left(\mu_{\lambda,+}\right)$, and $t_{0}:=\widehat{\mu}_{\lambda}\left(\sigma_{0}\right)$ and by equation (17) $\chi_{N_{\lambda}^{\lambda}} \mathrm{d} \nu_{\lambda}=\mathrm{d} \lambda^{\lambda}$ and $\chi_{N_{\tau}^{\lambda}} \mathrm{d} \nu_{\lambda}=\mathrm{d} \tau^{\lambda}$, gives us a $t$-scale-based impulsive differential equation:

$$
\begin{aligned}
x_{\lambda}(t) & =\xi_{0}+\int_{\sigma_{0}}^{\mu_{\lambda,+}(t)} h^{\nu}\left(\mu_{\lambda,-}(v), x_{\lambda}(v)\right) \mathrm{d} \nu_{\lambda}^{\nu} \\
& =\xi_{0}+\int_{t_{0}}^{t} h^{\nu}\left(\mu_{\lambda,+}(s), x_{\lambda}(s)\right) \mathrm{d} \nu^{\lambda} \\
& =\xi_{0}+\int_{t_{0}}^{t}(\underbrace{f^{v}\left(\mu_{\lambda,+}(s), x_{\lambda}(s)\right) \mathrm{d} \lambda^{\lambda}}_{\text {absolute continuous }}+\underbrace{g^{\nu}\left(\mu_{\lambda,+}(s), x_{\lambda}(s)\right) \mathrm{d} \tau^{\lambda}}_{\text {singular }}), \quad \forall t \in\left[a, b_{\lambda}\right] .
\end{aligned}
$$

It is important to note that the $v$-scale concepts define a second impulsive system with $\tau$.

2.3.2. The $\tau$-Scale from $\nu$-Scale. We show now that $\left[a, b_{\nu}\right], N_{\lambda}^{v}, N_{\tau}^{v}$, and $\nu$ determine $\left[a, b_{\tau}\right], N_{\lambda}^{\tau}, N_{\tau}^{\tau}, \lambda_{\tau}$, and $\tau_{\tau}$.
As stated in Section 2.3.1, the characteristic functions of $N_{\lambda}^{v}$ and $N_{\tau}^{v}$ are $\nu$-integrable. Let the time scale interval be $\left[a, b_{\tau}\right]$ with $b_{\tau}:=a+\int_{a}^{b_{v}} \chi_{N_{\tau}^{\nu}} \mathrm{d} \nu$,

$$
\begin{array}{rlrl}
\widehat{\mu}_{\tau}(s) & :=a+\int_{a}^{s} \chi_{N_{\tau}^{v}} \mathrm{~d} \nu \in\left[a, b_{\tau}\right], & & \forall s \in\left[a, b_{\nu}\right], \\
\mu_{\tau,-}(s) & :=\inf \widehat{\mu}_{\tau}^{-1}(\{s\}) \in\left[a, b_{\nu}\right], & & \forall s \in\left[a, b_{\tau}\right], \\
\mu_{\tau}(s) & :=\mu_{\tau,+}(s):=\sup \widehat{\mu}_{\tau}^{-1}(\{s\}) \in\left[a, b_{\nu}\right], & & \forall s \in\left[a, b_{\tau}\right], \\
\widehat{\mu}_{\tau} \cdot \mu_{\tau,-}^{\tau}(t) & =\widehat{\mu}_{\tau} \cdot \mu_{\tau,-}^{\tau}(t)=i d_{\left[a, b_{\tau}\right]}, & \\
v^{\tau}(s) & =a+\int_{a}^{\mu_{\tau,+}^{\tau}(s)} 1 \mathrm{~d} \nu^{\nu}=a+\int_{a}^{\mu_{\tau,+}^{\tau}(s)}\left(\chi_{N_{\lambda}^{v}}+\chi_{N_{\tau}^{v}}\right) \mathrm{d} \nu^{\nu}=a+\lambda^{\tau}([a, s))+\tau^{\tau}([a, s)), & \forall s \in\left[a, b_{\tau}\right],
\end{array}
$$


where $v^{\tau}$ is right continuous.

Since $v^{\tau}$ is strictly ascending, hence bijective, $N_{\lambda}^{\tau}=$ $\left(\nu^{\tau}\right)^{-1}\left(N_{\lambda}^{\nu}\right), N_{\tau}^{\tau}=\left(\nu^{\tau}\right)^{-1}\left(N_{\tau}^{\nu}\right)$, and

$$
\lambda^{\tau}\left(N_{\tau}^{\tau}\right)=0=\tau^{\tau}\left(N_{\lambda}^{\tau}\right),
$$

which means $\tau^{\tau}$ is singular with respect to $\lambda^{\tau}$ and $\lambda^{\tau}$ is singular with respect to $\tau^{\tau}$.
$D_{\tau}:=\left\{t \mid \mu_{\tau,-}(t) \neq \mu_{\tau,+}(t), t \in\left[a, b_{\tau}\right]\right\}$ is the countable set of discontinuity points in $\tau$-scale. Hence, based on equation (22) with $h^{\nu}, x_{\tau}:=\varphi\left(\mu_{\tau,+}\right)$, and $\vartheta_{0}:=\widehat{\mu}_{\tau}\left(\sigma_{0}\right)$ and based on equations (17) and (27), $\chi_{N^{\tau}} \mathrm{d} \nu_{\tau}=\mathrm{d} \lambda^{\tau}$, and $\chi_{N_{\tau}^{\tau}} \mathrm{d} \nu_{\tau}=\mathrm{d} \tau^{\tau}$, gives us a $\tau$-scale-based impulsive differential equation:

$$
\begin{aligned}
x_{\tau}(\vartheta) & =\xi_{0}+\int_{\sigma_{0}}^{\mu_{\tau,+}(\vartheta)} h^{v}\left(\mu_{\tau,-}(v), x_{\tau}(v)\right) \mathrm{d} v_{\tau}^{v} \\
& =\xi_{0}+\int_{\vartheta_{0}}^{\vartheta} h^{v}\left(\mu_{\tau,+}(s), x_{\tau}(s)\right) \mathrm{d} v^{\tau} \\
& =\xi_{0}+\int_{\vartheta_{0}}^{\vartheta}(\underbrace{f^{v}\left(\mu_{\tau,+}(s), x_{\tau}(s)\right) \mathrm{d} \lambda^{\tau}}_{\text {singular }}+\underbrace{g^{v}\left(\mu_{\tau,+}(s), x_{\tau}(s)\right) \mathrm{d} \tau^{\tau}}_{\text {absolut continuous }}), \quad \forall \vartheta \in\left[a, b_{\tau}\right] .
\end{aligned}
$$

2.3.3. Interpretation of the Two Representations. We need an interpretation of the two representations (26) and (29) of the Bainovian impulsive systems. The impulsive process described by equations (1) and (4) is composed from a process with $f$-dynamics and a process with $g$-dynamics. In $t$-scale equation (26), the fully described process with $f$-dynamics (absolute continuous component) is exposed to impulses generated by the (singular) impulse generator with $g$-dynamics. The second $\tau$-scale representation equation (29) gives a full description of the (absolute continuous) impulse generator with $g$-dynamics while being exposed to the (singular) counter impacts caused by the process with $f$ dynamics. Thus, these systems operate in action process/ reaction-counter action processes, which is a deep principle in interactions in sciences.
The $v$-scale representation presents both processes in full details in a time-sharing system. The $\nu$-scale process is presented in equation (30). The $\nu$-scale process is absolute continuous and makes it possible to prove existence theorems using Caratheodory's techniques.

In our presentation, we use the model in equations (1), (4), and (10). The $f^{v}$-dynamics operates in intervals $\left[\mu_{\lambda,-}\left(t_{i}\right), \mu_{\lambda,+}\left(t_{i+1}\right)\right) \subset N_{\lambda}^{v}, \forall t_{i}, t_{i+1} \in D_{\lambda}$ while the $g^{v}$-dynamics operates in intervals $\left[\mu_{\lambda,-}\left(t_{i}\right), \mu_{\lambda,+}\left(t_{i}\right)\right) \subset N_{\tau}^{v}$, $\forall t_{i} \in D_{\lambda}$. Hence, the $\nu$-scale consists of connected intervals alternating between $f^{\nu}$-dynamics and $g^{\nu}$-dynamics. The actions of the $f^{v}$-dynamics and $g^{\nu}$-dynamics are as indicated by the column headings in equation (30). The impulse points selected are $t_{i}, t_{i+1}, t_{i+2}$, and $t_{i+3} \in D_{\lambda}, t_{i}<t_{i+1}<t_{i+2}<t_{i+3}$ :

$$
\begin{array}{rllll} 
& f^{v}, \chi_{N_{\tau}^{v}}=0, \tau=\text { const. } & & g^{\nu}, \chi_{N_{\lambda}^{v}}=0, t=\text { const. } \\
\ldots \quad\left[\mu_{\lambda,+}^{\lambda}\left(t_{i}\right), \mu_{\lambda,-}^{\lambda}\left(t_{i+1}\right)\right) & \longrightarrow & {\left[\mu_{\lambda,-}^{\lambda}\left(t_{i+1}\right), \mu_{\lambda,+}^{\lambda}\left(t_{i+1}\right)\right)} & \longrightarrow \\
& {\left[\mu_{\lambda,+}^{\lambda}\left(t_{i+1}\right), \mu_{\lambda,-}^{\lambda}\left(t_{i+2}\right)\right)} & \longrightarrow & {\left[\mu_{\lambda,-}^{\lambda}\left(t_{i+1}\right), \mu_{\lambda,+}^{\lambda}\left(t_{i+2}\right)\right)} & \longrightarrow \\
& {\left[\mu_{\lambda,+}^{\lambda}\left(t_{i+2}\right), \mu_{\lambda,-}^{\lambda}\left(t_{i+3}\right)\right)} & \longrightarrow & {\left[\mu_{\lambda,-}^{\lambda}\left(t_{i+2}\right), \mu_{\lambda,+}^{\lambda}\left(t_{i+3}\right)\right)} & \cdots \\
& t_{i}, t_{i+1}, t_{i+2}, t_{i+3} \in D_{\lambda} .
\end{array}
$$

This scheme of operation follows the rules of timed chess game. The players are $f^{v}$-dynamics and $g^{\nu}$-dynamics. Their clocks are $t$ and $\tau$, respectively. If a solution of equations (1) and (4) is $\varphi:\left[t_{i}, t_{i}+\delta\right) \longrightarrow \Omega$ with a suitable $\delta>t_{i+3}-t_{i}$, then $f^{v}$ plays on the interval $\left[\mu_{\lambda,+}^{\lambda}\left(t_{i}\right), \mu_{\lambda,-}^{\lambda}\left(t_{i+1}\right)\right)$ for a period of $t_{i+1}-t_{i} t$-time while $g^{v}$ waiting with stopped $\tau$-time till $f^{v}$ produces $\varphi\left(t_{i+1}-0\right)$. Actions of $f^{v}$ are unknown to $g^{\nu}$. At $t_{i+1}$ the game switches to $g^{\nu}$, the $t$-clock stops, $\tau$-clock operates, and $g^{v}$ performs its job for a period of $\mu_{\lambda,+}^{\lambda}\left(t_{i+1}\right)-\mu_{\lambda,-}^{\lambda}\left(t_{i+1}\right)$ of $\tau$-time. The actions of $g^{\nu}$ are not known by $f^{\nu}$. This continues in this order until the solution exits. Note that the intervals are left-closed right-open by the fact that the new player starts to play at the leftmost point of its domain.

Therefore, in the $t$-scale process all $g$-actions are hidden and each $g$-interval appears in the form of jump. In the case of $\tau$-scale representation, all $f$-intervals appear as jumps. Hence, the two equations are completely symmetric and form a pair of impulsive differential equations.

2.3.4. Initial Value Problem for a Pair of Impulsive Differential Equations. From the interpretation of a pair of impulsive differential equation follows that the concept of 
initial value problem as presented in the Bainovian model, (1), (4), and (10) requires some clarifications. The Bainovian model discussed in Section 2.3.3 has form (4) or more likely (10) and the initial time $t_{0} \in\left[a, b_{\lambda}\right] \backslash D_{\lambda}$ cannot be a discontinuity point. From the analysis of equation (30) follows that any change between $f^{v} \longrightarrow g^{v}$ or $g^{v} \longrightarrow f^{v}$ takes place starting from the leftmost point of the domain/interval of the new dynamics. Hence, $\left[\mu_{\lambda_{+}}^{\lambda}\left(t_{i}\right), \mu_{\lambda_{-}-}^{\lambda}\left(t_{i+1}\right)\right) \subset N_{\lambda}^{v}$ is in the domain of $f^{\nu}$ and $\left[\mu_{\lambda,-}^{\lambda}\left(t_{i+1}\right), \mu_{\lambda,+}^{\lambda}\left(t_{i+1}\right)\right) \subset N_{\tau}^{v}$ is in the domain of $g^{v}$ in the $\left[t_{i}, t_{i+1}\right]$ interval, $\forall t_{i}, t_{i+1} \in D_{\lambda}$. Therefore, $\forall \sigma_{0} \in\left[a, b_{\nu}\right]$ either $\sigma_{0} \in N_{\lambda}^{\nu} \Rightarrow \widehat{\mu}_{\lambda}\left(\sigma_{0}\right)=t_{0} \in N_{\lambda}^{\lambda} \subset\left[a, b_{\lambda}\right]$ or $\sigma_{0} \in N_{\tau}^{v} \Rightarrow \widehat{\mu}_{\tau}\left(\sigma_{0}\right)=\vartheta_{0} \in N_{\tau}^{\tau} \subset\left[a, b_{\tau}\right]$.

Using the left closed right open intervals in equation (30) we obtain a pair of impulsive differential equations such that any initial value problem $\left(\sigma_{0}, \xi\right) \in\left[a, b_{\gamma}\right] \times \Omega_{0}, \varphi\left(\sigma_{0}\right)=\xi$ has a solution on an interval $\left[\sigma_{0}, \sigma_{0}+\delta_{0}\right) \subset N_{\lambda}^{v}$ if $\sigma_{0} \in N_{\lambda}^{v}$ or has a solution on an interval $\left[\sigma_{0}, \sigma_{0}+\delta_{0}\right) \subset N_{\tau}^{v}$ if $\sigma_{0} \in N_{\tau}^{v}$. Finally, an initial value problem will give initial value problems on the $t$-scale and on the $\tau$-scale as follows. Since
TABLE 1: The mappings in the first column are strictly ascending left and right continuous versions of $v^{\lambda}$ while the mapping in the second column is absolute continuous and ascending.

\begin{tabular}{lll}
\hline$\left[a, b_{\lambda}\right] \longrightarrow\left[a, b_{\nu}\right]$ & {$\left[a, b_{\lambda}\right] \longleftrightarrow\left[a, b_{\nu}\right]$} & Relation: \\
$\mu_{\lambda,-}^{\lambda}$ & {$\left[a, b_{\nu}\right] \longrightarrow\left[a, b_{\lambda}\right]$} & $\widehat{\mu}_{\lambda} \cdot \mu_{\lambda,-}^{\lambda}=i d_{\left[a, b_{\lambda}\right]}$ \\
$\mu_{\lambda}^{\lambda}:=\mu_{\lambda,+}^{\lambda}$ & $\widehat{\mu}_{\lambda}$ & $\widehat{\mu}_{\lambda} \cdot \mu_{\lambda,+}^{\lambda}=i d_{\left[a, b_{\lambda}\right]}$ \\
\hline
\end{tabular}

the solution is $x_{\lambda}=\varphi \cdot \mu_{\lambda,+}$ and $x_{\tau}=\varphi \cdot \mu_{\tau,+}$, we simply can define the initial value problems as follows:

$$
\begin{aligned}
\sigma_{0} \in N_{\lambda}^{\nu}: \Rightarrow t_{0}:=\widehat{\mu}_{\lambda}\left(\sigma_{0}\right) \text { and } \sigma_{0}=\mu_{\lambda,+}\left(t_{0}\right) ; \xi_{0, \lambda}:=\varphi\left(\mu_{\lambda,+}\left(t_{0}\right)\right)=\xi_{0} \\
\vartheta_{0}:=\widehat{\mu}_{\tau}\left(\sigma_{0}\right) ; \xi_{0, \tau}:=\varphi\left(\mu_{\tau,+}\left(\vartheta_{0}\right)\right) \text { hence let } \sigma_{0, \tau}:=\mu_{\tau,+}\left(\vartheta_{0}\right) .
\end{aligned}
$$

Similarly, for the case $\sigma_{0} \in N_{\tau}^{\nu}$,

$$
\begin{aligned}
\sigma_{0} \in N_{\tau}^{\nu}: \vartheta_{0}:=\widehat{\mu}_{\tau}\left(\sigma_{0}\right) \text { and } \sigma_{0}=\mu_{\tau,+}\left(\vartheta_{0}\right) \Rightarrow \xi_{0, \tau}:=\varphi\left(\mu_{\tau,+}\left(\vartheta_{0}\right)\right)=\xi_{0} \\
t_{0}:=\widehat{\mu}_{\lambda}\left(\sigma_{0}\right) ; \xi_{0, \lambda}:=\varphi\left(\mu_{\lambda,+}\left(t_{0}\right)\right) \text { hence let } \sigma_{0, \lambda}:=\mu_{\lambda,+}\left(t_{0}\right) .
\end{aligned}
$$

2.3.5. Integral Transformations among $t$-, $\tau$-, and $\nu$-Scales. The details of the assertions in this section come from paper [10].

We summarize the mappings between $t$-scale to $v$-scale listed in Section 2.3.1 (Table 1).

The mappings $\tau^{\lambda}$, and $\nu^{\lambda}$ are not continuous ascending functions, with a common set of discontinuity points $D_{\lambda}$. Therefore, the measures $\tau^{\lambda}$ and $\nu^{\lambda}$ are defined on the semiring $\mathscr{P}_{\nu,\left[a, b_{\gamma}\right], c}=\left\{[s, t) \mid s, t \in\left[a, b_{\lambda}\right] \backslash D_{\lambda}, s \leq t\right\}$ and the measures $\tau^{\lambda}([s, t)):=\tau^{\lambda}(t)-\tau^{\lambda} \quad(s)$ and $\nu^{\lambda}[(s, t)):=v^{\lambda}$ $(t)-\nu^{\lambda}(s), \forall[s, t) \in \mathscr{P}_{\nu},\left[a, b_{\lambda}\right], c$ can be extended to $\mathscr{B}_{\nu,\left[a, b_{\lambda}\right], c}=\sigma\left(\mathscr{P}_{\nu,\left[a, b_{\lambda}\right], c}\right)$.

The mappings $\mu_{\lambda,-}^{\lambda}, \mu_{\lambda,+}^{\lambda}:\left[a, b_{\lambda}\right] \longrightarrow\left[a, b_{\nu}\right]$ map the set of discontinuity points $D_{\lambda}$ into the set of left-closed rightopen intervals:

$$
\mathscr{D}_{\lambda}^{\nu}:=\left\{\left[\mu_{\lambda,-}(t), \mu_{\lambda,+}(t)\right) \mid t \in D_{\lambda}\right\},
$$

and the set of discontinuity points in $\left[a, b_{\nu}\right]$ is

$$
D_{\lambda}^{v}:=\underset{t \in D_{\lambda}}{\cup}\left[\mu_{\lambda,-}(t), \mu_{\lambda,+}(t)\right) \subset\left[a, b_{\nu}\right] .
$$

Moreover, the mappings $\mu_{\lambda,-}^{\lambda}, \mu_{\lambda,+}^{\lambda}$ are bijective on the set of continuity points $\left[a, b_{\nu}\right] \backslash D_{\lambda}^{\gamma}$ and $\mu_{\lambda,-}(t)=\mu_{\lambda,+}(t)$, $\forall t \in\left[a, b_{\lambda}\right] \backslash D_{\lambda}$.

The mappings $\mu_{\lambda,-}^{\lambda}, \mu_{\lambda,+}^{\lambda}:\left[a, b_{\lambda}\right] \longrightarrow\left[a, b_{\nu}\right]$ transform $[s, t) \in \mathscr{P}_{\nu,\left[a, b_{\lambda}\right], c} \longrightarrow\left[\nu^{\lambda}(s), \nu^{\lambda}(t)\right)=\left[\mu_{\lambda,-}^{\lambda}(s), \mu_{\lambda,-}^{\lambda}(t)\right)=$ $\left[\mu_{\lambda,+}^{\lambda}(s), \mu_{\lambda,+}^{\lambda}(t)\right) \in \mathscr{P}_{\nu,\left[a, b_{\gamma}\right], c}^{v}$ by their continuity points with $\mathscr{P}_{\nu,\left[a, b_{\gamma}\right], c}^{\nu}:=\left\{[s, t) \mid s, t \in\left[a, b_{\nu}\right] \backslash D_{\lambda}^{\nu}\right\}$.

The measure $\nu_{\lambda}^{\nu}$ on $\mathscr{P}_{\nu,\left[a, b_{v}\right], c}$ is defined by $v_{\lambda}^{\nu}([u, v)):=v-u=\mu_{\lambda,-}\left(\widehat{\mu}_{\lambda}^{\lambda}(v)\right)-\mu_{\lambda,-}\left(\widehat{\mu}_{\lambda}^{\lambda}(u)\right)=v_{\lambda}\left(\widehat{\mu}_{\lambda}^{\lambda}([u\right.$, $v))) \forall[u, v) \in \mathscr{P}_{\nu,\left[a, b_{v}\right], c}^{v}$ Also, if $[s, t) \in \mathscr{P}_{\nu,\left[a, b_{\lambda}\right], c}$, then $\left[\mu_{\lambda,-}(s), \mu_{\lambda,-}(t)\right) \in \mathscr{P}_{\nu,\left[a, b_{\nu}\right], c}^{v} \quad$ and $\quad v_{\lambda}^{\nu}\left(\left[\mu_{\lambda,-}(s), \mu_{\lambda,-}(t)\right)\right)=$ $\mu_{\lambda,-}(t)-\mu_{\lambda,-}(s)=\nu_{\lambda}([s, t))$. Let the smallest $\sigma$-algebra containing the semiring $\mathscr{P}_{\lambda,\left[a, b_{\nu}\right], c}^{\nu}$ be $\mathscr{B}_{\lambda,\left[a, b_{\gamma}\right], c}^{\nu}:=$ $\sigma\left(\mathscr{P}_{\lambda,\left[a, b_{\gamma}\right], c}^{\nu}\right)$ with the extended measure $\nu_{\lambda}^{\nu}$ on it.

From this follows that if $h:\left[a, b_{\nu}\right] \longrightarrow R$ is $\mathscr{B}_{\lambda,\left[a, b_{\nu}\right], c}^{\nu}$-measurable and $\nu_{\lambda}^{\nu}$-integrable, then

$$
\int_{a}^{\mu_{\lambda,+}^{\lambda}{ }^{(t)}} h \mathrm{~d} \nu_{\lambda}^{\nu}=\int_{a}^{t} h \cdot \mu_{\lambda,-}^{\lambda} \mathrm{d} \nu_{\lambda}, \quad \forall t \in\left[a, b_{\lambda}\right] .
$$

Conversely, if $h:\left[a, b_{\lambda}\right] \longrightarrow R$ is $\mathscr{B}_{\lambda,\left[a, b_{\nu}\right], c}$-measurable and $\nu_{\lambda}$-integrable, then

$$
\int_{a}^{\sigma} h \cdot \widehat{\mu}_{\lambda} \mathrm{d} \nu_{\lambda}^{\nu}=\int_{a}^{\widehat{\mu}_{\lambda}(\sigma)} h \mathrm{~d} \nu_{\lambda}, \quad \forall \sigma \in\left[a, b_{\nu}\right] \backslash D_{\lambda}^{\nu} .
$$

These are some of the main conclusions from paper [10] presented here in a condensed form.

We summarize the mappings between $\tau$-scale and $\nu$-scale listed in Section 2.3.2 (Table 2):

The mappings $\lambda^{\tau}$ and $v^{\tau}$ are not continuous ascending functions, with a common set of discontinuity points $D_{\tau}$. Therefore, the measures $\lambda^{\tau}$ and $\nu^{\tau}$ are defined on the semiring $\mathscr{P}_{\nu,\left[a, b_{\tau}\right], c}=\left\{[s, t) \mid s, t \in\left[a, b_{\tau}\right] \backslash D_{\tau}, s \leq t\right\}$ and the measures $\lambda^{\tau}([s, t)):=\lambda^{\tau}(t)-\lambda^{\tau}(s)$ and $\nu^{\tau}([s, t)):=v^{\tau}(t)-$ $\nu^{\tau}(s), \forall[s, t) \in \mathscr{P}_{\nu,\left[a, b_{\tau}\right], c}$ can be extended to $\mathscr{B}_{\nu,\left[a, b_{\tau}\right], c}=$ $\sigma\left(\mathscr{P}_{\nu,\left[a, b_{\tau}\right], c}\right)$.

The mappings $\mu_{\tau,-}^{\tau}, \mu_{\tau,+}^{\tau}:\left[a, b_{\tau}\right] \longrightarrow\left[a, b_{\gamma}\right]$ map the set of discontinuity points $D_{\tau}$ into the set of left-closed right-open intervals:

$$
\mathscr{D}_{\tau}^{\nu}:=\left\{\left[\mu_{\tau,-}(t), \mu_{\tau,+}(t)\right) \mid t \in D_{\tau}\right\}
$$

and the set of discontinuity points in $\left[a, b_{\nu}\right]$ is 
TABLE 2: The mappings in the first column are strictly ascending left and right continuous versions of $v^{\tau}$ while the mapping in the second column is absolute continuous and ascending.

\begin{tabular}{lll}
\hline$\left[a, b_{\tau}\right] \longleftrightarrow\left[a, b_{\gamma}\right]$ & {$\left[a, b_{\tau}\right] \longleftrightarrow\left[a, b_{\gamma}\right]$} & Relation : \\
$\mu_{\tau,-}^{\tau}$ & {$\left[a, b_{\gamma}\right] \longrightarrow\left[a, b_{\tau}\right]$} & $\widehat{\mu}_{\tau} \cdot \mu_{\tau,-}^{\tau}=i d_{\left[a, b_{\tau}\right]}$ \\
$\mu_{\tau}^{\tau}:=\mu_{\tau,+}^{\tau}$ & $\widehat{\mu}_{\tau}$ & $\widehat{\mu}_{\tau} \cdot \mu_{\tau,+}^{\tau}=i d_{\left[a, b_{\tau}\right]}^{\tau}$ \\
\hline
\end{tabular}

$$
D_{\tau}^{v}:=\bigcup_{t \in D_{\tau}}\left[\mu_{\tau,-}(t), \mu_{\tau,+}(t)\right) \subset\left[a, b_{\nu}\right]
$$

Moreover, the mappings $\mu_{\tau,-}^{\tau}$ and $\mu_{\tau,+}^{\tau}$ are bijective on the set of continuity points $\left[a, b_{\nu}\right] \backslash D_{\tau}^{\nu}$ and $\mu_{\tau,-}(t)=\mu_{\tau,+}(t)$, $\forall t \in\left[a, b_{\tau}\right] \backslash D_{\tau}$.

The mappings $\mu_{\tau,-}^{\tau}, \mu_{\tau,+}^{\tau}:\left[a, b_{\tau}\right] \longrightarrow\left[a, b_{\gamma}\right]$ transform $[s, t) \in \mathscr{P}_{\nu,\left[a, b_{\tau}\right], c} \longrightarrow\left[\nu^{\tau}(s), \nu^{\tau}(t)\right)=\left[\mu_{\tau,-}^{\tau}(s), \mu_{\tau,-}^{\tau}(t)\right)=$ $\left[\mu_{\tau,+}^{\tau}(s), \mu_{\tau,+}^{\tau}(t)\right) \in \mathscr{P}_{\nu,\left[a, b_{\gamma}\right], c}^{\nu}$ by their continuity points with $\mathscr{P}_{\nu,\left[a, b_{\gamma}\right], c}^{\nu}:=\left\{[s, t) \mid s, t \in\left[a, b_{\nu}\right] \backslash D_{\lambda}^{\nu}\right\}$.

The measure $\nu_{\tau}^{v}$ on $\mathscr{P}_{\nu,\left[a, b_{\gamma}\right], c}^{v}$ is defined by $\nu_{\tau}^{v}([u, v)):=$ $v-u=\mu_{\tau,-}\left(\widehat{\mu}_{\tau}^{\tau}(v)\right)-\mu_{\tau,-}\left(\widehat{\mu}_{\tau}^{\tau}(u)\right)=v_{\tau}\left(\hat{\mu}_{\tau}^{\tau}([u, v))\right) \forall[u, v)$ $\in \mathscr{P}_{\nu,\left[a, b_{v}\right], c}$. Also, if $[s, t) \in \mathscr{P}_{\nu,\left[a, b_{\tau}\right], c}$ then $\left[\mu_{\tau,-}(s), \mu_{\tau,-}(t)\right)$ $\in \mathscr{P}_{\nu,\left[a, b_{v}\right], c}^{v}$ and $v_{\tau}^{\nu}\left(\left[\mu_{\tau,-}(s), \mu_{\tau,-}(t)\right)\right)=\mu_{\tau,-}(t)-\mu_{\tau,-}(s)=$ $\nu_{\tau}([s, t))$. Let the smallest $\sigma$-algebra containing the semiring $\mathscr{P}_{\tau,\left[a, b_{\nu}\right], c}^{v}$ be $\mathscr{B}_{\tau,\left[a, b_{\nu}\right], c}^{\nu}:=\sigma\left(\mathscr{P}_{\tau,\left[a, b_{v}\right], c}^{\nu}\right)$ with the extended measure $v_{\tau}^{v}$ on it.

From this follows that if $h:\left[a, b_{\gamma}\right] \longrightarrow R$ is $\mathscr{B}_{\tau,\left[a, b_{\nu}\right], c}^{v}$-measurable and $v_{\tau}^{v}$-integrable, then

$$
\int_{a}^{\mu_{\tau,+}^{\tau}(t)} h \mathrm{~d} \nu_{\tau}^{\nu}=\int_{a}^{t} h \cdot \mu_{\tau,-}^{\tau} \mathrm{d} \nu_{\tau}, \forall t \in\left[a, b_{\tau}\right] .
$$

Conversely, if $h:\left[a, b_{\tau}\right] \longrightarrow R$ is $\mathscr{B}_{\tau,\left[a, b_{\nu}\right], c}$-measurable and $\nu_{\tau}$-integrable, then

$$
\int_{a}^{\sigma_{0}} h \cdot \widehat{\mu}_{\tau} \mathrm{d} \nu_{\tau}^{\nu}=\int_{a}^{\widehat{\mu}_{\tau}\left(\sigma_{0}\right)} h \mathrm{~d} \nu_{\tau}, \quad \forall \sigma \in\left[a, b_{\nu}\right] \backslash D_{\tau}^{\nu} .
$$

These are some of the main conclusions of paper [10] presented here in a condensed form.

The details about measures and Radon-Nikodym derivatives summarised below are in paper [10].

Let $\mathscr{B}\left(\left[a, b_{\gamma}\right], v\right)$ denote the Borel sets on $\left[a, b_{\nu}\right]$, and let $\nu$ be the Lebesgue measure on the $\sigma$-algebra $\mathscr{B}\left(\left[a, b_{\nu}\right], v\right)$.
Then,

$\mathscr{B}_{\lambda,\left[a, b_{\nu}\right], c}^{\nu} \subset \mathscr{B}\left(\left[a, b_{\nu}\right], v\right)$ and $\mathscr{B}_{\tau,\left[a, b_{\nu}\right], c}^{\nu} \subset \mathscr{B}\left(\left[a, b_{\nu}\right], \nu\right)$.

Let $f:\left[a, b_{\nu}\right] \longrightarrow R^{n}$ be a $\mathscr{B}\left(\left[a, b_{\gamma}\right], \nu\right)$-measurable $\nu$-integrable function. Let $\nu_{f, \lambda}(A):=\int_{A} f \mathrm{~d} \nu, \forall A \in \mathscr{B}_{\lambda,\left[a, b_{\nu}\right], c}^{\nu}$ and $\nu_{f, \tau}(A):=\int_{A} f \mathrm{~d} \nu, \forall A \in \mathscr{B}_{\tau,\left[a, b_{\gamma}\right], c}$ be signed measures absolute continuous with respect to the measures $\nu_{\lambda}^{\nu}$ and $\nu_{\tau}^{\nu}$, respectively. Then, their Radon-Nikodym derivatives with respect to $\nu_{\lambda}^{v}$ and $\nu_{\tau}^{v}$ give

$$
\begin{aligned}
\widehat{f}_{\nu, \lambda} & :=\frac{\mathrm{d} v_{f, \lambda}}{\mathrm{d} \nu_{\lambda}^{\nu}} \Longleftrightarrow \int_{A} f \mathrm{~d} \nu \\
& =\int_{A} \widehat{f}_{\nu, \lambda} \mathrm{d} \nu_{\lambda}^{\nu}, \quad \forall A \in \mathscr{\mathscr { B }}_{\lambda,\left[a, b_{\nu}\right], c}^{v}, \widehat{f}_{\nu, \lambda} \in L_{1}\left(\nu_{\lambda}, \mathscr{B}_{\lambda,\left[a, b_{\nu}\right], c}^{\nu}\right),
\end{aligned}
$$

$$
\begin{aligned}
\hat{f}_{\nu, \tau} & :=\frac{\mathrm{d} v_{f, \tau}}{\mathrm{d} \nu_{\tau}^{\nu}} \Longleftrightarrow \int_{A} f \mathrm{~d} \nu \\
& =\int_{A} \hat{f}_{\nu, \tau} \mathrm{d} \nu_{\tau}^{\nu}, \quad \forall A \in \mathscr{B}_{\tau,\left[a, b_{\nu}\right], c}^{\nu}, \hat{f}_{\nu, \tau} \in L_{1}\left(\nu_{\tau}^{\nu}, \mathscr{B}_{\tau,\left[a, b_{\nu}\right], c}^{\nu}\right) .
\end{aligned}
$$

Combining equations (35) and (41) gives

$$
\int_{a}^{\mu_{\lambda,+}^{\lambda}(t)} f \mathrm{~d} \nu=\int_{a}^{\mu_{\lambda,+}^{\lambda}(t)} \widehat{f}_{\nu, \lambda} \mathrm{d} \nu_{\lambda}^{\nu}=\int_{a}^{t} \widehat{f}_{\nu, \lambda} \cdot \mu_{\lambda,-}^{\lambda} \mathrm{d} \nu_{\lambda}, \quad \forall t \in\left[a, b_{\lambda}\right] .
$$

Combining equations (39) and (42) gives

$$
\int_{a}^{\mu_{\tau,+}^{\tau}(\vartheta)} f \mathrm{~d} \nu=\int_{a}^{\mu_{\tau,+}^{\tau}(\vartheta)} \widehat{f}_{\nu, \tau} d \nu_{\tau}^{\nu}=\int_{a}^{\vartheta} \widehat{f}_{\nu, \tau} \cdot \mu_{\tau,-}^{\tau} \mathrm{d} \nu_{\tau}, \quad \forall \vartheta \in\left[a, b_{\tau}\right] .
$$

It was proved in paper [40] that the Radon-Nikodym derivatives $\widehat{f}_{\nu, \lambda}$ and $\widehat{f}_{\nu, \tau}$ fulfill the relations:

$$
\begin{array}{ll}
f(s)=\hat{f}_{\nu, \lambda}(s), & \text { a.e.s } \in\left[a, b_{\nu}\right] \backslash D_{\lambda}^{\nu}, \\
f(s)=\hat{f}_{\nu, \tau}(s), & \text { a.e.s } \in\left[a, b_{\nu}\right] \backslash D_{\tau}^{v},
\end{array}
$$

which implies the assertions below as follow ups of the indicated equation (45):

$$
\begin{aligned}
& \int_{a}^{\mu_{\lambda,+}^{\lambda}(t)} f \times \chi_{\left[a, b_{\nu}\right] \backslash \mathscr{D}_{\lambda}^{\nu}} \mathrm{d} v=\int_{a}^{\mu_{\lambda,+}^{\lambda}(t)} \hat{f}_{\nu, \lambda} \times \chi_{\left[a, b_{\nu}\right] \backslash \mathscr{D}_{\lambda}^{\nu}} \mathrm{d} \nu_{\lambda}^{\nu}=\int_{(43)}^{\mu_{\lambda,+}^{\lambda}(t)} f \times \chi_{\left[a, b_{\nu}\right] \backslash \mathscr{D}_{\lambda}^{\nu}} \mathrm{d} \nu_{\lambda}^{\nu} \\
& =\int_{a}^{t}\left(f \times \chi_{\left[a, b_{\lambda}\right] \backslash \mathscr{D}_{\lambda}}\right) \cdot \mu_{\lambda,-}^{\lambda} \mathrm{d} \nu_{\lambda}, \quad \forall t \in\left[a, b_{\lambda}\right], \\
& \int_{a}^{\mu_{\tau,+}^{\tau}(9)} f \times \chi_{\left[a, b_{\nu}\right] \backslash \mathscr{D}_{\tau}^{\nu}} \mathrm{d} v=\int_{a}^{\mu_{\tau,+}^{\tau}(9)} \hat{f}_{\nu, \tau} \times \chi_{\left[a, b_{\nu}\right] \backslash \mathscr{D}_{\tau}^{\nu}} \mathrm{d} \nu_{\tau}^{\nu}=\int_{(43)}^{\mu_{\tau,+}^{\tau}(9)} f \times \chi_{\left[a, b_{\nu}\right] \backslash \mathscr{D}_{\tau}^{\nu}} \mathrm{d} \nu_{\tau}^{\nu} \\
& =\int_{a}^{\vartheta}\left(f \times \chi_{\left[a, b_{\tau}\right] \backslash \mathscr{D}_{\tau}}\right) \cdot \mu_{\tau,-}^{\tau} \mathrm{d} \nu_{\tau}, \quad \forall \vartheta \in\left[a, b_{\tau}\right] .
\end{aligned}
$$


This relation enables us to transform the solution of a $\nu$ scale differential equation into solutions of a $t$-scale $/ \tau$-scale impulsive differential equations.

The function $f$ can be written as $f=f \times \chi_{\left[a, b_{y}\right] \backslash \mathscr{D}^{v}}+f \times$ $\chi_{\mathscr{D}_{\lambda}^{\nu}}$ by $\left[a, b_{\nu}\right]=\left(\left[a, b_{\nu}\right] \backslash \mathscr{D}_{\lambda}^{\nu}\right) \cup \mathscr{D}_{\lambda}^{\nu}$ and $\varnothing=\left(\left[a, b_{\nu}\right] \backslash \mathscr{D}_{\lambda}^{\nu}\right) \cap$
Similarly, $\quad f=f \times \chi_{\left[a, b_{\nu}\right] \backslash \mathscr{D}_{\tau}^{\nu}}+f \times \chi_{\mathscr{D}_{\tau}^{\nu}} \quad$ by $\quad\left[a, b_{\nu}\right]=$ $\left(\left[a, b_{\nu}\right] \backslash \mathscr{D}_{\tau}^{\nu}\right) \cup \mathscr{D}_{\tau}^{\nu}$ and $\varnothing=\left(\left[a, b_{\gamma}^{\tau}\right] \backslash \mathscr{D}_{\tau}^{\nu}\right) \cap \mathscr{D}_{\tau}^{\nu}$.

Therefore, equations (46) and (47) can be rewritten as

$$
\begin{aligned}
& \int_{a}^{\mu_{\lambda,+}^{\lambda}{ }^{(t)}} f \mathrm{~d} \nu=\int_{a}^{\mu_{\lambda,+}^{\lambda}(t)}\left(f \times \chi_{\left[a, b_{\nu}\right] \backslash \mathscr{D}_{\lambda}^{\nu}}+f \times \chi_{\mathscr{D}_{\lambda}^{\nu}}\right) \mathrm{d} \nu=\int_{a}^{t}\left(f \times \chi_{\left[a, b_{\lambda}\right] \backslash \mathscr{D}_{\lambda}}+\left(\widehat{f \times \chi_{\mathscr{D}_{\lambda}^{\nu}}}\right)_{\nu, \lambda}\right) \cdot \mu_{\lambda,-}^{\lambda} \mathrm{d} \nu_{\lambda}, \quad \forall t \in\left[a, b_{\lambda}\right], \\
& \int_{a}^{\mu_{\lambda,+}^{\lambda}(t)} f \mathrm{~d} \nu=\int_{a}^{\mu_{\tau,+}^{\tau}{ }^{(9)}}\left(f \times \chi_{\left[a, b_{\nu}\right] \backslash \mathscr{D}_{\tau}^{\nu}}+f \times \chi_{\mathscr{D}_{\tau}^{\nu}}\right) \mathrm{d} \nu=\int_{a}^{\vartheta}\left(f \times \chi_{\left[a, b_{\tau}\right] \backslash \mathscr{D}_{\tau}}+\left(f \widehat{f \times \chi_{\mathscr{D}_{\tau}^{\nu}}}\right)_{\nu, \tau}\right), \quad \forall \vartheta \in\left[a, b_{\tau}\right] .
\end{aligned}
$$

The sets in $D_{\lambda}^{v}$ and in $D_{\tau}^{\nu}$ are atoms as described in Lemma 2.9 in [10], in detail. Since an atom in $\mathscr{B}_{\lambda,\left[a, b_{\nu}\right], c}^{\nu}$ does not have any proper measurable subset in $\mathscr{B}_{\lambda,\left[a, b_{\gamma}\right], c}^{\nu}$ but the same set is a nonatomic measurable set in $\mathscr{B}\left(\left[a, b_{\nu}\right], v\right), f$ can be integrated on it by $\nu$. Similarly, an atom in $\mathscr{B}_{\tau,\left[a, b_{v}\right], c}^{v}$ does not have any proper measurable subset in $\mathscr{B}_{\tau,\left[a, b_{\gamma}\right], c}^{v}$ but the same set is a nonatomic measurable set in $\mathscr{B}\left(\left[a, b_{\nu}\right], v\right)$, and $f$ can be integrated on it by $\nu$. Hence, $\forall t_{j} \in D_{\lambda}$ the set $\left[\mu_{\lambda,-}\left(t_{j}\right), \mu_{\lambda,+}\left(t_{j}\right)\right) \in D_{\lambda}^{\nu}$ is an atom. Similarly, $\forall \tau_{j} \in D_{\tau}$ the set $\left[\mu_{\tau,-}\left(\tau_{j}\right), \mu_{\tau,+}\left(\tau_{j}\right)\right) \in D_{\tau}^{v}$ is an atom. Therefore, the Radon-Nikodym derivatives in equation (48) can be written as follows:

$$
\begin{aligned}
& \left(\widehat{f \times \chi_{D_{\lambda}^{\nu}}}\right)_{\nu, \lambda}\left(t_{j}\right):=\frac{\int_{\mu_{\lambda,-}}^{\mu_{\lambda,+}\left(t_{j}\right)} f \mathrm{~d} \nu}{\mu_{\lambda,+}\left(t_{j}\right)-\mu_{\lambda,-}\left(t_{j}\right)}, \\
& \left({\widehat{f \times \chi_{D_{\tau}^{\nu}}}}_{\tau_{\nu, \tau}}\left(\tau_{j}\right):=\frac{\int_{\mu_{\tau,-}}^{\mu_{\tau,+}\left(\tau_{j}\right)} f \mathrm{~d} \nu}{\mu_{\tau,+}\left(\tau_{j}\right)-\mu_{\tau,-}\left(\tau_{j}\right)} .\right.
\end{aligned}
$$

2.4. Existence of the Solutions on $t$-scale and on $\tau$-Scale. In this section, we want to formulate the existence theorem for the pair of impulsive differential equations on the $t$-scale and $\tau$-scale.

First, we assume that Corollary 2 is true. The initial value problem in equation (21) has a solution $\varphi$ : $\left[\sigma_{0}, \sigma_{0}+\right.$ $\beta) \longrightarrow \mathscr{R}_{\delta, \varepsilon}\left(\sigma_{0}, \xi\right), \varphi\left(\sigma_{0}\right)=\xi$.
Applying the statements in equations (43) and (44) to equation (21), we get that the solution in $t$-scale can be $x_{\lambda}(t)=\varphi \cdot \mu_{\lambda,+}^{\lambda}(t), t \in\left[t_{0}, \widehat{\mu}_{\lambda}\left(\sigma_{0}+\beta\right)\right), t_{0}=\widehat{\mu}_{\lambda}\left(\sigma_{0}\right)$ and in $\tau$-scale it can be $x_{\tau}(\vartheta)=\varphi \cdot \mu_{\tau,+}^{\tau}(\vartheta), \vartheta \in\left[\vartheta_{0}, \widehat{\mu}_{\tau}\left(\sigma_{0}+\beta\right)\right)$, $\vartheta_{0}=\widehat{\mu}_{\tau}\left(\sigma_{0}\right)$, which transformed $\nu$-scale solutions. We have to prove that they fulfill the initial value problems with respect to the $t$-scale and $\tau$-scale versions and that also they fulfill the respective impulsive differential equations.

The initial value problem $\varphi\left(\sigma_{0}\right)=\xi$ on the $\nu$-scale problem fulfills either $\sigma_{0} \in N_{\lambda}^{\nu}$, which is detailed out in equation (31), or it fulfills $\sigma_{0} \in N_{\tau}^{v}$, which is detailed out in (32). Hence, the two cases of initial value problems fulfilled by the pair of impulsive differential equations are as follows:

When $\sigma_{0} \in N_{\lambda}^{\nu}, t_{0}:=\widehat{\mu}_{\lambda}\left(\sigma_{0}\right)$ and $\xi_{0, \lambda}:=\varphi\left(\mu_{\lambda,+}\left(t_{0}\right)\right)=\xi$ and $\vartheta_{0}:=\widehat{\mu}_{\tau}\left(\sigma_{0}\right)$ and $\xi_{0, \tau}:=\varphi\left(\mu_{\tau,+}\left(\vartheta_{0}\right)\right)$

When $\sigma_{0} \in N_{\tau}^{\nu}, \vartheta_{0}:=\widehat{\mu}_{\tau}\left(\sigma_{0}\right)$ and $\xi_{0, \tau}:=\varphi\left(\mu_{\tau,+}\left(\vartheta_{0}\right)\right)=\xi$ while $t_{0}:=\widehat{\mu}_{\lambda}\left(\sigma_{0}\right)$ and $\xi_{0, \lambda}:=\varphi\left(\mu_{\lambda,+}\left(t_{0}\right)\right)$

Note that the following equations (31) and (32) for the initial value problems are given in $t$-scale uniformly $\left(t_{0}, \xi_{0, \lambda}\right)$ and similarly for $\tau$-scale uniformly $\left(\vartheta_{0}, \xi_{0, \tau}\right)$, but the values are obtained differently according to equations (31) and (32) (the difference is whether $\xi_{0, \lambda}=\xi$ or $\xi_{0, \tau}=\xi$ ).

In these equations, we used that $\mu_{\lambda,+}: N_{\lambda}^{\lambda} \longrightarrow N_{\lambda}^{\nu}$ and $\mu_{\tau,+}: N_{\tau}^{\tau} \longrightarrow N_{\tau}^{\nu}$ which are bijective strictly ascending mappings. Hence, the initial conditions are fulfilled.

Solution of the pair of impulsive differential equations: let us apply relations (43) and (44) to solution (21) both on $t$ scale and on $\tau$-scale with $h^{\nu}$ :

$$
\begin{aligned}
\varphi\left(\mu_{\lambda,+}^{\lambda}(t)\right) & =\xi_{0}+\int_{\sigma_{0}}^{\mu_{\lambda,+}^{\lambda}(t)} h^{\nu}(v, \varphi(v)) \mathrm{d} \nu=\xi_{0}+\int_{\sigma_{0}}^{\mu_{\lambda,+}^{\lambda}(t)} \widehat{h^{v}}{ }_{\nu, \lambda}(v) \mathrm{d} \nu_{\lambda}^{v} \\
& =\xi_{0, \lambda}+\int_{t_{0}}^{t} \widehat{h^{v}}{ }_{\nu, \lambda}\left(\mu_{\lambda,-}^{\lambda}\right) \mathrm{d} \nu_{\lambda}, \quad \forall t \in\left[a, b_{\lambda}\right], \\
\varphi\left(\mu_{\tau,+}^{\tau}(\vartheta)\right) & =\xi_{0}+\int_{\sigma_{0}}^{\mu_{\tau,+}^{\tau}(9)} h^{\nu}(v, \varphi(v)) \mathrm{d} v=\xi_{0}+\int_{\sigma_{0}}^{\mu_{\tau,+}^{\tau}(\vartheta)} \widehat{h}_{\nu, \tau}^{v}(v) \mathrm{d} \nu_{\tau}^{v} \\
& =\xi_{0, \tau}+\int_{\vartheta_{0}}^{\vartheta} \widehat{h^{v}}{ }_{\nu, \tau}\left(\mu_{\tau,-}^{\tau}\right) \mathrm{d} \nu_{\tau}, \quad \forall \vartheta \in\left[a, b_{\tau}\right] .
\end{aligned}
$$


Let us split $h^{v}$ on $v$-scale with $\mathscr{D}_{\lambda}^{v}$ into a component on continuity points $h_{\lambda, c}^{\nu}:=h^{v} \times \chi_{\left[a, b_{\nu}\right] \backslash \mathscr{D}_{\lambda}^{\nu}}$ and a component on discontinuity points $h_{\lambda, d}^{\nu}:=h^{\nu} \times \chi_{\mathscr{D}^{\nu}}$. We can do this with discontinuity points $\mathscr{D}_{\tau}^{\nu}$ on $\nu$-scale also. A component on continuity points is $h_{\tau, c}^{v}:=h^{v} \times \chi_{\left[a, b_{v}\right] \backslash \mathscr{D}_{\tau}^{v}}$, and a component on discontinuity points is $h_{\tau, d}^{\nu}:=h^{\nu} \times \chi_{\mathscr{D}_{\tau}^{\nu}}$ :

$$
\begin{aligned}
& h^{v}=h_{\lambda, c}^{v}+h_{\lambda, d}^{v}, \\
& h^{v}=h_{\tau, c}^{v}+h_{\tau, d}^{v} .
\end{aligned}
$$

Let us put the definition $h^{v}:=f^{v} \chi_{N_{\lambda}^{v}}+g^{v} \chi_{N_{\tau}^{v}}$ into equation (52):

$$
\begin{aligned}
& h^{\nu}:=\left(f^{v} \chi_{N_{\lambda}^{v}}+g^{v} \chi_{N_{\tau}^{v}}\right) \times \chi_{\left[a, b_{\nu}\right] \backslash \mathscr{D}_{\lambda}^{\nu}}+\left(f^{v} \chi_{N_{\lambda}^{v}}+g^{\nu} \chi_{N_{\tau}^{v}}\right) \times \chi_{D_{\lambda}^{\nu}}, \\
& h^{\nu}:=\left(f^{v} \chi_{N_{\lambda}^{v}}+g^{\nu} \chi_{N_{\tau}^{v}}\right) \times \chi_{\left[a, b_{\nu}\right] \backslash \mathscr{D}_{\tau}^{\nu}}+\left(f^{\nu} \chi_{N_{\lambda}^{\nu}}+g^{\nu} \chi_{N_{\tau}^{v}}\right) \times \chi_{\mathscr{D}_{\tau}^{\nu}} .
\end{aligned}
$$

Considering $\quad N_{\lambda}^{\nu} \subset\left[a, b_{\nu}\right] \backslash \mathscr{D}_{\lambda}^{\nu}$ and $N_{\tau}^{\nu} \subset\left[a, b_{\nu}\right] \mathscr{D}_{\tau}^{\nu}$, $N_{\lambda}^{\nu} \cap N_{\tau}^{\nu}=\varnothing$ and $\mathscr{D}_{\lambda}^{v} \subset N_{\tau}^{v}$ and $\mathscr{D}_{\tau}^{\nu} \subset N_{\lambda}^{\nu}$ leads to

$$
\begin{aligned}
& h^{v}:=\underbrace{f^{v} \chi_{N_{\lambda}^{v}}+g^{v} \chi_{N_{\tau}^{v}} \times \chi_{\left[a, b_{v}\right] \backslash \mathscr{D}_{\lambda}^{v}}}_{h_{\lambda, c}^{v}}+\underbrace{g^{v} \times \chi_{\mathscr{D}_{\lambda}^{v}}}_{h_{\lambda, d}^{v}}, \\
& h^{v}:=\underbrace{f^{v} \chi_{N_{\lambda}^{v}} \times \chi_{\left[a, b_{\nu}\right] \backslash \mathscr{D}_{\tau}^{v}}+g^{v} \chi_{N_{\tau}^{v}}}_{h_{\tau, c}^{v}}+\underbrace{f^{v} \times \chi_{\mathscr{D}_{\tau}^{v}}}_{h_{\tau, d}^{v}} .
\end{aligned}
$$

Let us apply the relations in equations (50) and (51) to $h_{\lambda, d}^{v}$ and $h_{\tau, d}^{v}$, as expressed in equation (54) (cases of discontinuity):

$$
\begin{aligned}
& \int_{\sigma_{0}}^{\mu_{\lambda,+}^{\lambda}(t)} h_{\lambda, d} \mathrm{~d} v=\int_{\sigma_{0}}^{\mu_{\lambda,+}^{\lambda}(t)} g^{v} \times \chi_{\mathscr{D}_{\lambda}^{\nu}}(v, \varphi(v)) \mathrm{d} v \\
& \left.=\int_{\sigma_{0}}^{\mu_{\lambda,+}^{\lambda}(t)}\left(g^{\nu} \times \chi_{\mathscr{D}_{\lambda}^{\nu}}\right)_{\nu, \lambda}(v) \times \chi_{\left[a, b_{\nu}\right]}\right] \mathscr{D}_{\lambda}^{\nu} \mathrm{d} \nu_{\lambda}^{\nu}=\int_{t_{0}}^{t}\left(g^{\nu} \times \chi_{\mathscr{D}_{\lambda}^{\nu}}\right)_{\nu, \lambda} \cdot \mu_{\lambda,-}^{\lambda} \mathrm{d} \nu_{\lambda}, \quad \forall t \in\left[a, b_{\lambda}\right], \\
& \int_{\sigma_{0}}^{\mu_{\lambda,+}^{\lambda}(t)} h_{\tau, d} \mathrm{~d} \nu=\int_{\sigma_{0}}^{\mu_{\tau,+}^{\tau}(\vartheta)} f^{\nu} \times \chi_{\mathscr{D}_{\tau}^{\nu}}(\nu, \varphi(v)) \mathrm{d} \nu=\int_{\sigma_{0}}^{\mu_{\tau,+}^{\tau}(\vartheta)}\left(f^{\nu} \times \widehat{\chi_{D_{\tau}^{\nu}}}\right)_{\nu, \tau}(v) \times \chi_{\left[a, b_{\nu}\right] \backslash \mathscr{D}_{\tau}^{\nu}} \mathrm{d} \nu_{\tau}^{\nu} \\
& =\int_{\vartheta_{0}}^{\vartheta}\left(f^{\nu} \widehat{\times \chi_{D_{\tau}^{\nu}}}\right)_{\nu, \tau} \cdot \mu_{\tau,-}^{\tau} \mathrm{d} \nu_{\tau}^{\nu}, \quad \forall \vartheta \in\left[a, b_{\tau}\right]
\end{aligned}
$$

Let us apply equations (46) and (47) to the components $h_{\lambda, c}$ and $h_{\tau, c}$ using equation (54):

$$
\begin{aligned}
& \int_{\sigma_{0}}^{\mu_{\lambda,+}^{\lambda}(t)} h_{\lambda, c}^{\nu}(v, \varphi(v)) \mathrm{d} v=\int_{\sigma_{0}}^{\mu_{\lambda,+}^{\lambda}(t)} \widehat{h}_{\nu, \lambda}^{v}(v) \times \chi_{\left[a, b_{\nu}\right] \backslash \mathscr{D}_{\lambda}^{\nu}} \mathrm{d} \nu_{\lambda}^{\nu}=\int_{(43)}^{\mu_{\lambda,+}^{\lambda}{ }_{\sigma_{0}}^{(t)}} h_{\lambda, c}^{\nu}(v, \varphi(v)) \mathrm{d} \nu_{\lambda}^{\nu} \\
& =\int_{t_{0}}^{t}\left(f^{v} \chi_{N_{\lambda}^{v}} \cdot \mu_{\lambda,-}^{\lambda}+g^{\nu} \chi_{N_{\tau}^{v}} \times \chi_{\left[a, b_{\nu}\right] \backslash D_{\lambda}^{\nu}} \mu_{\lambda,-}^{\lambda}\right) \mathrm{d} \nu_{\lambda}, \quad \forall t \in\left[a, b_{\lambda}\right] \text {, } \\
& \int_{\sigma_{0}}^{\mu_{\tau,+}^{\tau}(9)} h_{\tau, \mathcal{c}}^{\nu}(v, \varphi(v)) \mathrm{d} \nu=\int_{\sigma_{0}}^{\mu_{\tau,+}^{\tau}(9)} \widehat{h_{\tau, c \nu, \tau}^{\nu}}(v) \times \chi_{\left[a, b_{\nu}\right] \backslash \mathscr{D}_{\tau}^{\nu}} \mathrm{d} \nu_{\tau}^{\nu}={ }_{(43)} \int_{\sigma_{0}}^{\mu_{\tau,+}^{\tau}(9)} h_{\tau, c}^{\nu}(v, \varphi(v)) \mathrm{d} \nu_{\tau}^{\nu} \\
& =\int_{\vartheta_{0}}^{\vartheta}\left(f^{\nu} \chi_{N_{\lambda}^{\nu}} \times \chi_{\left[a, b_{\nu}\right] \backslash \mathscr{D}_{\tau}^{\nu}} \mu_{\tau,-}^{\tau}+g^{\nu} \chi_{N_{\tau}^{\nu}} \circ \mu_{\tau,-}^{\tau}\right) \mathrm{d} \nu_{\tau}, \quad \forall \vartheta \in\left[a, b_{\tau}\right] \text {, }
\end{aligned}
$$

where equation (45) is obtained from Corollary 2 and Theorem 1 in [40].

The Radon-Nikodym derivatives of $h_{\lambda, d}^{v}$ and $h_{\lambda, d}^{\nu}$, remains to be determined. The domains of the Radon-Nikodym derivatives are countable unions of pairwise disjoint atoms of the $\sigma$-algebras $\mathscr{B}_{\lambda,\left[a, b_{v}\right], c}^{v}$ and $\mathscr{B}_{\tau,\left[a, b_{1}\right], c}^{v}$, respectively, as defined in equations: $\mathscr{D}_{\lambda}^{\nu}$ in (11); $\mathscr{D}_{\tau}^{\nu}$ in (37). The lists of these atoms are defined: $D_{\lambda}^{\nu}$ in (12) and $D_{\tau}^{\nu}$ in (38). This means that the Radon-Nikodym derivatives are fully determined on $\mathscr{D}_{\lambda}^{\nu}$ if they are determined on the intervals in $D_{\lambda}^{\nu}$, and they are fully determined on $\mathscr{D}_{\tau}^{\nu}$ if they are determined on each interval in $D_{\tau}^{\nu}$.

Let impulse time points $t_{j} \in D_{\lambda}$ and $\tau_{j} \in D_{\tau}$ be selected, and let $\varphi$ : $\left[t_{0}, t_{0}+\beta\right) \longrightarrow B_{\varepsilon_{0}}\left(x_{0}\right)$ be the solution of equation (21).

Based on equation (54), $h_{\lambda, d}^{v}$ and $h_{\tau, d}^{v}$ is replaced by $g^{v} \times \chi_{D_{\lambda}^{v}}$ and $f^{v} \times \chi_{D_{\tau}^{v}}$, respectively, in equation (49). This leads to 


$$
\begin{aligned}
& \left(\hat{g}^{\nu} \times \chi_{\mathscr{D}_{\lambda}^{\nu}}\right)_{\nu, \lambda}\left(t_{j}, \varphi\right):=\frac{\int_{\mu_{\lambda,-}\left(t_{j}\right)}^{\mu_{\lambda,+}\left(t_{j}\right)} g^{\nu} \mathrm{d} \nu}{\mu_{\lambda,+}\left(t_{j}\right)-\mu_{\lambda,-}\left(t_{j}\right)}=\frac{\varphi\left(\mu_{\lambda,+}^{\lambda}\left(t_{j}\right)\right)-\varphi\left(\mu_{\lambda,-}^{\lambda}\left(t_{j}\right)\right)}{\mu_{\lambda,+}^{\lambda}\left(t_{j}\right)-\mu_{\lambda,-}^{\lambda}\left(t_{j}\right)}=: \widehat{g}_{0 \lambda}^{\nu}\left(t_{j}, \varphi\right), \\
& \forall t_{j} \in D_{\lambda},
\end{aligned}
$$

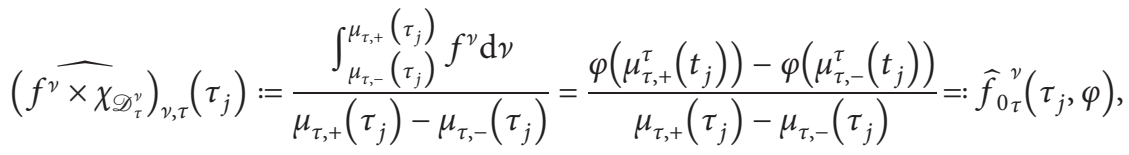

$$
\begin{aligned}
& \forall \tau_{j} \in D_{\tau} .
\end{aligned}
$$

Let $\mathscr{T}\left(\left[a, b_{\gamma}\right]\right)$ denote all the solution trajectories with domains as subsets of $\left[a, b_{\gamma}\right]$.

With equations (59) and (60), $\widehat{g}_{0 \lambda}^{\nu}$ is defined on the interval $\left[\mu_{\lambda,-}^{\lambda}\left(t_{j}\right)\right),\left[\mu_{\lambda,+}^{\lambda}\left(t_{j}\right)\right), \forall t_{j} \in D_{\lambda}$ and $\widehat{f}_{0 \lambda}^{\nu}$ is defined on the interval $\left[\mu_{\tau_{-}}^{\tau}\left(\tau_{j}\right)\right),\left[\mu_{\tau_{+}}^{\tau}\left(\tau_{j}\right)\right), \forall t_{j} \in D_{\tau^{\prime}}$; hence, $\widehat{g}_{0 \lambda}^{\nu}$ is defined on $\mathscr{D}_{\lambda}^{\nu}$ and $\hat{f}_{0 \tau}^{\nu}$ is defined on $\mathscr{D}_{\tau}^{\nu}$. Then, we define $\hat{g}_{\lambda}^{v} \& \hat{f}_{\tau}^{\nu}$ as follows:

$$
\begin{aligned}
& \widehat{g}_{\lambda}^{\nu}(\sigma, \varphi):=\left\{\begin{array}{ll}
\widehat{g}_{0 \lambda}^{\nu}(\sigma, \varphi), & \widehat{\mu}_{\lambda}(\sigma) \in D_{\lambda}, \\
0, & \sigma \notin \mathscr{D}_{\lambda}^{\nu},
\end{array} \quad \forall(\sigma, \varphi) \in\left[a, b_{\nu}\right] \times \mathscr{T}\left(\left[a, b_{\nu}\right]\right),\right. \\
& \hat{f}_{\tau}^{\nu}(\sigma, \varphi):= \begin{cases}\hat{f}_{0 \tau}^{\nu}(\sigma, \varphi), & \widehat{\mu}_{\tau}(\sigma) \in D_{\tau}, \\
0, & \sigma \notin \mathscr{D}_{\tau}^{\nu},\end{cases}
\end{aligned}
$$

These jumps in this case are dependent on the solution and the time parameter is based on $\left[a, b_{\gamma}\right]$. If however, the right sides fulfill Lipschitz condition then the solution is determined by $\left.t_{j}, \varphi\left(t_{j}\right)\right)$; hence, $\widehat{g}_{\lambda}\left(t_{j}, \varphi\right)$ and $\widehat{f}_{\tau}\left(\tau_{j}, \varphi\right)$ are fully determined by the initial value problems $\varphi\left(t_{j}\right)=$ $y,\left(t_{j}, y\right) \in\left\{t_{j}\right\} \times \Omega_{0}$ and $\varphi\left(\tau_{j}\right)=y,\left(\tau_{j}, y\right) \in\left\{\tau_{j}\right\} \times \Omega_{0}$; hence, the jumps can be written $\hat{g}_{\lambda}\left(t_{j}, \varphi\right) \longrightarrow \hat{g}_{\lambda}\left(t_{j}, \varphi\left(t_{j}\right)\right)$ and $\widehat{f}_{\tau}\left(\tau_{j}, \varphi\left(\tau_{j}\right)\right) \longrightarrow \widehat{f}_{\tau}\left(\tau_{j}, \varphi\left(\tau_{j}\right)\right)$ which is Bainov's formulation. Combining equations (55) and (57)equation and (56) about $h_{\lambda, c}^{v}$ and $h_{\tau, c}^{v}$ with $h_{\lambda, d}^{v}$ and $h_{\tau, d}^{v}$ and equations (59) and (60) on the Radon-Nikodym derivatives results in equations

$$
\begin{aligned}
& \varphi\left(\mu_{\lambda,+}^{\lambda}(t)\right)=\xi_{0}+\int_{\sigma_{0}}^{\mu_{\lambda,+}^{\lambda}(t)}\left(h_{\lambda, c}^{\nu}+h_{\lambda, d}^{\nu}\right)(v, \varphi(v)) \mathrm{d} v \\
& =\xi_{0, \lambda}+\int_{t_{0}}^{t}\left(f^{v}\left(\mu_{\lambda,-}^{\lambda}, \varphi\left(\mu_{\lambda,-}^{\lambda}\right)\right) \chi_{N_{\lambda}^{\lambda}}+g^{\nu}\left(\mu_{\lambda,-}^{\lambda}, \varphi\left(\mu_{\lambda,-}^{\lambda}\right)\right) \chi_{N_{\tau}^{\lambda}} \times \chi_{\left[a, b_{\lambda}\right] \mathscr{D}_{\lambda}}\right) \mathrm{d} \nu_{\lambda} \\
& +\int_{t_{0}}^{t} \widehat{g}_{\lambda}^{\nu}\left(\mu_{\lambda,-}^{\lambda}, \varphi\right) \mathrm{d} v_{\lambda}, \quad \forall t \in\left[a, b_{\lambda}\right] \\
& \varphi\left(\mu_{\tau,+}^{\tau}(\vartheta)\right)=\xi_{0}+\int_{\sigma_{0}}^{\mu_{\tau,+}^{\tau}(9)}\left(h_{\tau, c}^{\nu}+h_{\tau, d}^{\nu}\right)(v, \varphi(v)) \mathrm{d} v \\
& =\xi_{0, \tau}+\int_{\vartheta_{0}}^{\vartheta}\left(f^{\nu}\left(\mu_{\tau,-}^{\tau}, \varphi\left(\mu_{\tau,-}^{\tau}\right)\right) \chi_{N_{\lambda}^{\tau}} \times \chi_{\left[a, b_{\tau}\right] \backslash \mathscr{D}_{\tau}}+g^{\nu}\left(\mu_{\tau,-}^{\tau}, \varphi\left(\mu_{\tau,-}^{\tau}\right) \chi_{N_{\tau}^{\tau}}\right)\right) \mathrm{d} \nu_{\tau} \\
& +\int_{\vartheta_{0}}^{\vartheta} \widehat{f}_{\tau}^{v}\left(\mu_{\tau,-}^{\lambda}, \varphi\right) \mathrm{d} v_{\tau}, \quad \forall \vartheta \in\left[a, b_{\tau}\right] .
\end{aligned}
$$


Let us put into equations (62) and (63) the expressions $x_{\lambda}:=\varphi\left(\mu_{\lambda,-}^{\lambda}\right)$ and $x_{\tau}:=\varphi\left(\mu_{\tau,-}^{\tau}\right)$, and from equation (17) $\chi_{N_{\lambda}^{\lambda}} \mathrm{d} \nu_{\lambda}=\mathrm{d} \lambda$, and $\chi_{N_{\tau}^{\tau}} \mathrm{d} \nu_{\tau}=\mathrm{d} \tau$. This leads to

$$
\begin{aligned}
x_{\lambda}(t)= & \xi_{0, \lambda}+\int_{t_{0}}^{t} \underbrace{f^{\nu}\left(\mu_{\lambda,-}^{\lambda}, x_{\lambda}\right) \mathrm{d} \lambda^{\lambda}}_{\text {absolute continuous }} \\
& +\int_{t_{0}}^{t}(\underbrace{g^{\nu}\left(\mu_{\lambda,-}^{\lambda}, x_{\lambda}\right) \times \chi_{\left[a, b_{\lambda}\right] \backslash \mathscr{D}_{\lambda}}}_{\text {continuous singular }}+\underbrace{\hat{g}_{\lambda}\left(\mu_{\lambda,-}^{\lambda}, \varphi\right)}_{\text {pure jumping singular }}) \mathrm{d} \tau^{\lambda}, \quad \forall t \in\left[a, b_{\lambda}\right], \\
x_{\tau}(\vartheta)= & \xi_{0, \tau}+\int_{\vartheta_{0}}^{\vartheta} \underbrace{g^{\nu}\left(\mu_{\tau,-}^{\tau}, x_{\tau}\right) \mathrm{d} \tau^{\tau}}_{\text {absolute continuous }} \\
& +\int_{\vartheta_{0}}^{\vartheta}(\underbrace{f^{\nu}\left(\mu_{\tau,-}^{\tau}, x_{\tau}\right) \times \chi_{\left[a, b_{\tau}\right] \backslash \mathscr{D}_{\tau}}}_{\text {continuous singular }}+\underbrace{\hat{f}_{\tau}\left(\mu_{\tau,-}^{\tau}, \varphi\right)}_{\text {pure jumping singular }}) \mathrm{d} \lambda^{\tau}, \quad \forall \vartheta \in\left[a, b_{\tau}\right] .
\end{aligned}
$$

Conclusion 2. We proved that, from the statement of Corollary 2, follows the existence of the solution of the pair of impulsive differential equations. Important to note that the function $f$ is fully known in $t$-scale and in $\nu$-scale, whereas the function $g$ is fully known in $\tau$-scale and $\nu$-scale. Therefore, we will prove that if $f^{\lambda}$ is locally $t$-integrable $\forall(t, x) \in\left[a, b_{\lambda}\right] \times \Omega_{0}$ and $g^{\tau}$ is locally $\tau$-integrable $\forall(\vartheta, x) \in$ $\left[a, b_{\tau}\right] \times \Omega_{0}$, then the corresponding $h^{\nu}:\left[a, b_{\nu}\right] \longrightarrow R^{n}$ is locally $\nu$-integrable $\forall(\sigma, x) \in\left[a, b_{\gamma}\right] \times \Omega_{0}$ (see the definition of the concept of local t-integrability in Definition 1 ).

Lemma 1. Let $[a, b] \subset R$ be a closed bounded interval and let $\psi:[a, b] \times \Omega_{0} \longrightarrow R^{n}$ be measurable in its variable $t \in[a, b]$ for any fixed $x \in \Omega_{0}$, and let it be continuous in its variable $x \in \Omega_{0}$ for each fixed $t \in[a, b]$. If $\psi$ is locally integrable at each $\left(t_{0}, x_{0}\right) \in[a, b] \times \Omega_{0}$ then there exists an $0<\varepsilon \in R$ and an integrable dominator $m_{x_{0}}:[a, b] \longrightarrow R^{+} \backslash\{0\}$ such that $\|\psi(t, x)\| \leq m_{x_{0}}(t), \forall(t, x) \in[a, b] \times B_{\varepsilon}\left(x_{0}\right) \subset[a, b] \times \Omega_{0}$, $\forall\left(t_{0}, x_{0}\right) \in[a, b] \times \Omega_{0}$.

Proof. Let $\psi$ be extended to 0 outside $[a, b] \subset R$. Let $\left(t_{0}, x_{0}\right) \in[a, b] \times \Omega_{0}$. By the formulation of the lemma $\exists \mathscr{R}_{\delta_{t_{0}, x_{0}}, \varepsilon_{t_{0}, x_{0}}}\left(t_{0}, x_{0}\right)$ and $m_{t_{0}, x_{0}}:\left(t_{0}-\delta_{t_{0}, x_{0}}, t_{0}+\delta_{t_{0}, x_{0}}\right) \longrightarrow R^{+}$ $\backslash\{0\}$ such that $\|\psi(t, x)\| \leq m_{t_{0}, x_{0}}(t), \forall(t, x) \in\left(t_{0}-\delta_{t_{0}, x_{0}}, t_{0}+\right.$ $\left.\delta_{t_{0}, x_{0}}\right) \times B_{\varepsilon_{t_{0}, x_{0}}}\left(x_{0}\right)$. Then, $[a, b] \subset \cup_{s \in[a, b]}\left(s-\delta_{s, x_{0}}, s+\delta_{s, x_{0}}\right)$. Since $[a, b]^{2}$ is compact, a finite subsystem $\left\{\left(t_{j}, x_{0}\right) \mid 1 \leq\right.$ $j \leq N\}$ can be selected such that $[a, b] \subset \cup_{j=1}^{N}\left(t_{j}-\delta_{t_{j}, x_{0}}, t_{j}+\right.$ $\left.\delta_{t_{j}, x_{0}}\right)$ covers the interval $[a, b]$. Extending $m_{t_{j}, x_{0}}$ by zero outside $\left(t_{j}-\delta_{t_{i}, x_{0}}, t_{j}+\delta_{t_{j}, x_{0}}\right), \forall 1 \leq j \leq N$, we can form a positive integrable dominator $m_{x_{0}}(t):=\max \left\{m_{t_{j}, x_{0}}(t) \mid 1 \leq\right.$ $j \leq N\}, \forall t \in[a, b]$, and can get a positive $\varepsilon_{x_{0}}:=\min \left\{\varepsilon_{t_{j}, x_{0}} \mid 1\right.$ $\leq j \leq N\}$ such that the positive dominator $m_{x_{0}}:[a, b] \longrightarrow R^{+} \backslash\{0\} \quad$ fulfills $\quad\|\psi(t, x)\| \leq m_{x_{0}}(t), \forall(t, x)$ $\in[a, b] \times B_{\varepsilon_{x_{0}}}\left(x_{0}\right) \subset[a, b] \times \Omega_{0}$.

Theorem for the existence of solutions of a pair of impulsive differential equations: let us denote the functions $f$ and $g$ on $t$-scale by $f^{\lambda}: N_{\lambda}^{\lambda} \longrightarrow R^{n}$ and $g^{\lambda}: N_{\tau}^{\lambda} \longrightarrow R^{n}$, on $\tau$-scale by $f^{\tau}: N_{\lambda}^{\tau} \longrightarrow R^{n}$ and $g^{\tau}: N_{\tau}^{\tau} \longrightarrow R^{n}$, and on $\nu$-scale by $f^{\nu}: N_{\lambda}^{\nu} \longrightarrow R^{n}$ and $g^{\nu}: N_{\tau}^{\nu} \longrightarrow R^{n}$. Let $h^{\nu}:=\left(f^{\lambda} \cdot \widehat{\mu}_{\lambda}\right)$ $\chi_{N_{\lambda}^{v}}+\left(g^{\tau} \cdot \widehat{\mu}_{\tau}\right) \chi_{N_{\tau}^{\nu}}:\left[a, b_{\nu}\right] \longrightarrow R^{n}$.

Lemma 2. The following statements are equivalent:

Statement 1: the mapping $f^{\lambda}$ is locally t-integrable $\forall(t, x) \in\left[a, b_{\lambda}\right] \times \Omega_{0}$ and the mapping $g^{\tau}$ is locally $\tau$-integrable $\forall(\vartheta, x) \in\left[a, b_{\tau}\right] \times \Omega_{0}$

Statement 2: the mapping $h^{\nu}$ is locally $v$-integrable $\forall(\sigma, x) \in\left[a, b_{\gamma}\right] \times \Omega_{0}$

Proof. Statement $1 \Rightarrow$ Statement 2:

(1) $f^{\lambda}$ is 1 Statement 2: the mapping $h^{\nu}$ is locally $\nu$-integrable $\forall(\sigma, x) \in\left[a, b_{\nu}\right] \times \Omega_{0}$ and locally $t$-integrable $\quad \forall\left(t_{0}, x_{0}\right) \in\left[a, b_{\lambda}\right] \times \Omega_{0} \Rightarrow \exists 0<\varepsilon_{\lambda, x_{0}} \in R$ and $m_{\lambda, x_{0}}:\left[a, b_{\lambda}\right] \longrightarrow R^{+} \backslash\{0\}$ such that $\| f^{\lambda}(t$, $x) \| \leq m_{\lambda, x_{0}}(t), \forall(t, x) \in\left[a, b_{\lambda}\right] \times B_{\varepsilon_{\lambda, x_{0}}}\left(x_{0}\right) \subset\left[a, b_{\lambda}\right]$ $\times \Omega_{0} \forall x_{0} \in \Omega_{0}$ by Lemma 1 .

(2) $g^{\tau}$ is locally $\tau$-integrable $\forall\left(\vartheta_{0}, x_{0}\right) \in\left[a, b_{\tau}\right] \times$ $\Omega_{0} \Rightarrow \exists 0<\varepsilon_{\tau, x_{0}} \in R$ and $m_{\tau, x_{0}}:\left[a, b_{\tau}\right] \longrightarrow R^{+} \backslash\{0\}$ such that $\left\|g^{\tau}(\vartheta, x)\right\| \leq m_{\tau, x_{0}}(\vartheta), \forall(\vartheta, x) \in\left[a, b_{\tau}\right] \times$ $B_{\varepsilon_{\tau, x_{0}}}\left(x_{0}\right) \subset\left[a, b_{\tau}\right] \times \Omega_{0}, \forall x_{0} \in \Omega_{0}$ by Lemma 1 .

(3) By point 1 in $\left[a, b_{\lambda}\right]$, let $x_{0} \in \Omega_{0}$; then, $\exists 0<\varepsilon_{\lambda, x_{0}} \in R$ such that $\left\|f^{\lambda}(t, x)\right\| \leq m_{\lambda, x_{0}}(t), \forall(t, x) \in\left[a, b_{\lambda}\right] \times$ $B_{\varepsilon_{\lambda, x_{0}}}\left(x_{0}\right) \subset\left[a, b_{\lambda}\right] \times \Omega_{0} \Rightarrow\left\|f^{\lambda}\left(\widehat{\mu}_{\lambda}(\sigma), x\right)\right\| \leq m_{\lambda, x_{0}}\left(\widehat{\mu}_{\lambda}\right.$ 
$(\sigma)), \forall(\sigma, x) \in\left[a, b_{\nu}\right] . \times B_{\varepsilon_{1, x_{0}}}\left(x_{0}\right) \subset\left[a, b_{\nu}\right] \times \Omega_{0}$.

Hence, with $f^{\nu}(\sigma, x):=f^{\lambda}\left(\widehat{\mu}_{\lambda}(\sigma), x\right) \quad \times \chi_{N^{v}}(\sigma)$, $\left\|f^{\nu}(\sigma, x)\right\| \leq m_{\lambda, x_{0}}\left(\widehat{\mu}_{\lambda}(\sigma)\right) \times \chi_{N_{\lambda}^{v}}(\sigma), \forall(\sigma, x) \in N_{\lambda}^{\nu} \times$ $B_{\varepsilon_{1, x_{0}}}\left(x_{0}\right) \subset\left[a, b_{v}\right] \times \Omega_{0}$.

(4) By point 1 in $\left[a, b_{\tau}\right]$, let $x_{0} \in \Omega_{0}$; then, $\exists 0<\varepsilon_{\tau, x_{0}} \in R$ such that $\left\|g^{\tau}(\vartheta, x)\right\| \leq m_{\tau, x_{0}}(\vartheta), \forall(\vartheta, x) \in\left[a, b_{\tau}\right] \times$ $B_{\varepsilon_{\tau}}\left(x_{0}\right) \subset\left[a, \quad b_{\tau}\right] \times \Omega_{0} \Rightarrow\left\|g^{\tau}\left(\widehat{\mu}_{\tau}(\sigma), x\right)\right\| \leq m_{\tau}$, $x_{0}\left(\mu_{\tau}(\sigma)\right), \quad \forall(\sigma, x), \in\left[a, b_{v}\right] \times B_{\varepsilon_{\tau, x}}\left(x_{0}\right) \subset\left[a, b_{v}\right] \times$ $\Omega_{0}$. Hence, with $g^{\nu}(\sigma, x):=g^{\tau}\left(\widehat{\mu}_{\tau}^{\tau},(\sigma), x\right) \times \chi_{N^{v}}(\sigma)$, $\left\|g^{\nu}(\sigma, x)\right\| \leq m_{\tau, x_{0}}\left(\widehat{\mu}_{\tau}(\sigma)\right) \times \chi_{N_{\tau}^{\nu}}(\sigma), \forall(\sigma, x) \in N_{\lambda}^{\bar{v}} \times$ $B_{\varepsilon_{\tau, x_{0}}}\left(x_{0}\right) \subset\left[a, b_{\gamma}\right] \times \Omega_{0}$.

(5) By point 3, $\left\|f^{v}(\sigma, x)\right\| \leq m_{\lambda, x_{0}}\left(\widehat{\mu}_{\lambda}(\sigma)\right) \times \chi_{N_{1}^{v}}(\sigma)$ in $N_{\lambda}^{v} \times B_{\varepsilon_{\lambda, x_{0}}}\left(x_{0}\right)$, and by point 4 , $\left\|g^{\nu}(\sigma, x)\right\| \leq$ $m_{\tau, x_{0}}\left(\hat{\mu}_{\tau}^{\lambda, x_{0}}(\sigma)\right) \times \chi_{N_{\tau}^{v}}(\sigma)$ hold in $N_{\lambda}^{v} \times B_{\varepsilon_{\tau, x_{0}}}\left(x_{0}\right)$. Then, with $\quad m_{v, x_{0}}(\sigma):=\max \left\{m_{\lambda, x_{0}}\left(\widehat{\mu}_{\lambda}(\sigma)\right) \times \chi_{N_{\lambda}^{v}}(\sigma)\right.$, $\left.m_{\tau, x_{0}}\left(\widehat{\mu}_{\tau}(\sigma)\right) \times \chi_{N_{\tau}^{\nu}}(\sigma)\right\}, \forall \sigma \in\left[a, b_{\nu}\right] \quad$ and with $\varepsilon_{v, x_{0}}:=\min \left\{\varepsilon_{\lambda, x_{0}}, \varepsilon_{\tau, x_{0}}\right\}$, we obtain that $\left\|h^{v}(\sigma, x)\right\|=$ $\left\|f^{\nu}(\sigma, x)+g^{\nu}(\sigma, x)\right\| \leq m_{\nu, x_{0}}(\sigma), \quad \forall(\sigma, x) \in\left[a, b_{\nu}\right] \times$ $B_{\varepsilon_{v, x_{0}}}\left(x_{0}\right)$.

Statement $\quad 2 \Rightarrow$ Statement $1: \quad$ assume that $\exists 0<\varepsilon_{\nu, x_{0}} \in R$ and $m_{\nu, x_{0}}:\left[a, b_{\gamma}\right] \longrightarrow R^{+} \backslash\{0\} \forall x_{0} \in \Omega_{0} \quad$ such that $\left\|h^{\nu}(\sigma, x)\right\| \leq m_{v, x_{0}}(\sigma) \forall(\sigma, x) \in\left[a, b_{\nu}\right] \times B_{\varepsilon_{v, x_{0}}}\left(x_{0}\right)$, where $h^{v}:=\left(f^{v} \chi_{N_{\lambda}^{v}}+g^{v} \chi_{N_{\tau}^{v}}\right):\left[a, b_{v}\right] \times \Omega_{0} \longrightarrow R^{n}$.

(1) $\left\|h^{v}(\sigma, x)\right\| \leq m_{\gamma, x_{0}}(\sigma) \Rightarrow\|\| h^{v}\left(\mu_{\lambda,-}(t), x\right) \| \leq m_{\gamma, x_{0}}\left(\mu_{\lambda,-}\right.$ $(t))$ holds $\forall(t, x) \in\left[a, b_{\lambda}\right] \times B_{\varepsilon_{v},}\left(x_{0}\right)$. Multiplying both sides by the characteristic function of $N_{\lambda}^{\lambda}$, we obtain that

$$
\begin{aligned}
\left\|h^{v}\left(\mu_{\lambda,-}(t), x\right) \chi_{N_{\lambda}^{\lambda}}\right\| & =\left\|\left(f^{v} \chi_{N_{\lambda}^{v}}+g^{v} \chi_{N_{\tau}^{v}}\right)\left(\mu_{\lambda,-}(t), x\right) \chi_{N_{\lambda}^{\lambda}}\right\| \\
& =\left\|\left(f^{v}\left(\mu_{\lambda,-}(t), x\right) \chi_{N_{\lambda}^{\lambda}}(t)+g^{v}\left(\mu_{\lambda,-}(t), x\right) \chi_{N_{\tau}^{\lambda}}(t)\right) \chi_{N_{\lambda}^{\lambda}}(t)\right\| \\
& =\left\|f^{v}\left(\mu_{\lambda,-}(t), x\right) \chi_{N_{\lambda}^{\lambda}}(t)\right\| \leq m_{v, x_{0}}\left(\mu_{\lambda,-}(t)\right) \forall(t, x) \in\left[a, b_{\lambda}\right] \times B_{\varepsilon_{v, x_{0}}}\left(x_{0}\right),
\end{aligned}
$$

which proves the case for the $t$-scale. The case for $\tau$-scale is word for word the same as the case of $t$-scale and is left to the reader.

Conclusion 3. With this we proved that the initial value problem prescribed for a pair of impulsive differential equations has a solution if Caratheodory's condition holds for $f^{\lambda}: N_{\lambda}^{\lambda} \longrightarrow R^{n}$ and for $g^{\tau}: N_{\tau}^{\tau} \longrightarrow R^{n}$. This condition is equivalent to the condition of Corollary 2 . We state a final version of the existence theorem.

\section{Condition 1}

C1: let $f^{\lambda}:(t, x) \in N_{\lambda}^{\lambda} \times \Omega_{0} \longrightarrow f^{\lambda} \quad(t, x) \in R^{n}$ and $g^{\tau}:(\vartheta, x) \in N_{\tau}^{\tau} \times \Omega_{0} \longrightarrow g^{\tau}(\vartheta, x) \in R^{n}$ be measurable functions of $t$ and $\vartheta$, for each fixed $x$, respectively, and let they be continuous in $x$ for each fixed $t$ and $\vartheta$, respectively, $\forall(t, x) \in N_{\lambda}^{\lambda} \times \Omega_{0}$ and $\forall(\vartheta, x) \in N_{\tau}^{\tau} \times \Omega_{0}$. C2: let $\quad f^{\nu}(\sigma, x):=f^{\lambda}\left(\widehat{\mu}_{\lambda}(\sigma), x\right)$ and $g^{\nu}(\sigma, x):=$ $g^{\tau}\left(\widehat{\mu}_{\tau}(\sigma), x\right) \forall(\sigma, x) \in\left[a, b_{\gamma}\right] \times \Omega_{0}$. Then, by condition $\mathrm{C} 1, \quad h^{\nu}(\sigma, x):=f^{\nu}(\sigma, x) \chi_{N_{\lambda}^{\nu}}(\sigma)+g^{\nu}(\sigma, x) \chi_{N_{\tau}^{\nu}}(\sigma)$ is measurable in $\sigma$ for each fixed $x$, and it is continuous in $x$ for each fixed $\sigma, \forall(\sigma, x) \in\left[a, b_{\gamma}\right] \times \Omega_{0}$.

C3: let $f^{\lambda}$ and $g^{\tau}$ be locally $t$ - and $\tau$-integrable on their respective domains or equivalently let $h^{\nu}$ be locally $\nu$-integrable on $\left[a, b_{v}\right] \times \Omega_{0}$.

Theorem 2. Let the mappings $f^{\lambda}, g^{\tau}$, and $h^{\nu}$ fulfill Condition $1 \mathrm{C} 1, \mathrm{C} 2$, and C3. Let $\left(\sigma_{0}, \xi_{0}\right) \in\left[a, b_{v}\right] \times \Omega_{0}$ be a fixed point and let a cylinder $\mathscr{R}_{\delta, \varepsilon}\left(\sigma_{0}, \xi_{0}\right) \subset\left[a, b_{\gamma}\right] \times \Omega_{0}$ exist with a D.I.F. $m:\left[\sigma_{0}, \sigma_{0}+\delta\right) \longrightarrow R^{+} \backslash\{0\}$ on $\mathscr{R}_{\delta, \varepsilon}\left(\sigma_{0}, \xi_{0}\right)$ to $h^{\nu}$. Then, there exists an interval $\left[\sigma_{0}, \sigma_{0}+\beta\right), 0<\beta \leq \delta$ such that equation (21) has a solution $\varphi$ in that interval such that $(\sigma, \varphi(\sigma)) \in \mathscr{R}_{\delta, \varepsilon}\left(\sigma_{0}, \xi\right), \forall \sigma \in\left[\sigma_{0}, \sigma_{0}+\beta\right)$ and $\varphi\left(\sigma_{0}\right)=\xi_{0}$.

Moreover, let $t_{0}=\widehat{\mu}_{\lambda}\left(\sigma_{0}\right) \in\left[a, b_{\lambda}\right]$ and $\vartheta_{0}=$ $\widehat{\mu}_{\tau}\left(\sigma_{0}\right) \in\left[a, b_{\lambda}\right] . \quad$ Let $\quad x_{\lambda}:=\varphi \cdot \mu_{\lambda,+}^{\lambda}:\left[t_{0}, \widehat{\mu}_{\lambda}\left(\sigma_{0}+\beta\right)\right) \longrightarrow$ $B_{\varepsilon}\left(\xi_{0}\right)$ and let $x_{\tau}:=\varphi \cdot \mu_{\tau,+}^{\tau}:\left[\vartheta_{0}, \widehat{\mu}_{\tau}\left(\sigma_{0}+\beta\right)\right) \longrightarrow B_{\varepsilon}\left(\xi_{0}\right)$. Let $x_{\lambda}\left(t_{0}\right):=\varphi\left(\mu_{\lambda,+}^{\lambda}\left(t_{0}\right)\right)=: \xi_{0, \lambda}$ and let $x_{\tau}\left(\vartheta_{0}\right):=\varphi\left(\mu_{\tau,+}^{\tau}\right.$ $\left.\left(\vartheta_{0}\right)\right)=\xi_{0, \tau}$. Then, the following equations hold:

$$
\begin{aligned}
x_{\lambda}(t)= & \xi_{0, \lambda}+\int_{t_{0}}^{t} f^{\lambda}\left(s, x_{\lambda}(s)\right) \mathrm{d} \lambda^{\lambda} \\
& +\int_{t_{0}}^{t}\left(g^{\tau}\left(\widehat{\mu}_{\tau}\left(\mu_{\lambda,-}^{\lambda}(s)\right), x_{\lambda}(s)\right) \times \chi_{\left[a, b_{\lambda}\right] \backslash \mathscr{D}_{\lambda}}+\widehat{g}^{\nu}\left(\mu_{\lambda,-}^{\lambda}(s)\right)\right) \mathrm{d} \tau^{\lambda}, \quad \forall t \in\left[t_{0}, \widehat{\mu}_{\lambda}\left(\sigma_{0}+\beta\right)\right), \\
x_{\tau}(\vartheta)= & \xi_{0, \tau}+\int_{\vartheta_{0}}^{\vartheta} g^{\tau}\left(\eta, x_{\tau}(\eta)\right) \mathrm{d} \tau^{\tau} \\
& +\int_{\vartheta_{0}}^{\vartheta}\left(f^{\lambda}\left(\widehat{\mu}_{\lambda}\left(\mu_{\tau,-}^{\tau}(\eta)\right), x_{\tau}(\eta)\right) \times \chi_{\left[a, b_{\tau} \mid \mathscr{D}_{\tau}\right]}+\widehat{f}^{\nu}\left(\mu_{\tau,-}^{\tau}(\eta)\right)\right) \mathrm{d} \lambda^{\tau}, \quad \forall \vartheta \in\left[\vartheta_{0}, \widehat{\mu}_{\lambda}\left(\sigma_{0}+\beta\right)\right) .
\end{aligned}
$$


Proof. In the theorem, $f^{\nu}(\sigma, x)=f^{\lambda}\left(\widehat{\mu}_{\lambda}(\sigma), x\right)$ and $g^{\nu}(\sigma, x)=g^{\tau}\left(\hat{\mu}_{\tau}(\sigma), x\right)$ by 2.1, C2. Then, $f^{\nu}\left(\mu_{\lambda,-}^{\lambda}(t), x\right)=$ $f^{\lambda}\left(\widehat{\mu}_{\lambda}\left(\mu_{\lambda,-}^{\lambda}(t)\right), x\right)=f^{\lambda}\left(i d_{\left[a, b_{\lambda}\right]}(t), x\right)=f^{\lambda}(t, x)$ and $g^{\nu}\left(\mu_{\tau,-}^{\tau}\right.$ $(\vartheta), x)=g^{\tau}\left(\widehat{\mu}_{\tau}\left(\mu_{\tau,-}^{\tau}(\vartheta)\right), x\right)=g^{\tau}\left(i d_{\left[a, b_{\tau}\right]}(t), x\right)=g^{\tau} \quad(\vartheta, x)$, where we applied the identities listed in equations (24) and (27).

Conclusion 4. The Bainovian impulsive processes describe the movements of a process ( $f$-dynamics) under the impulses of another process ( $g$-dynamics). In this paper, we developed a technique to split such a system into two impulsive processes. One is the Bainovian process moving with $f$ dynamics under the impulses of the process with $g$-dynamics, while the other is the process with $g$-dynamics under the impacts of $f$-impulses on it. The relationship is an action/reaction interaction between the two processes. This gives a pair of impulsive differential equation with the relationship between them analysed. We established an existence theorem for impulsive differential equations with right side being a measurable function of time which facilitates the analysis of delayed impulsive differential equations. On the contrary, trajectories may be connected together by impulse effects. This will make the flow of solutions to be a tree structure instead of a connected flow as in ordinary differential equations. The leaves of the tree consist of trajectories coming from discontinuity points without history. This tree is directed, with orientation from the leaves to the root which is called in-tree or antiarborescence [41]. This gives wide range of modelling facilities by enabling one to model and study mixing new generations in addition to studying flows of solutions.

\section{Data Availability}

The data used to support the findings of this study are included within the article.

\section{Conflicts of Interest}

The authors declare that they have no conflicts of interest.

\section{References}

[1] D. D. Bainov and P. S. Simeonov, Impulsive Differential Equations-Asymptotic Properties of the Solutions, World Scientific Pub. Coy. Pte. Ltd, Singapore, 1995.

[2] H. G. Ballinger, Qualitative theory of impulsive delay differential equations, Ph.D. thesis, University of Waterloo, Waterloo, Canada, 1999.

[3] V. Lakshmikantham, D. D. Bainov, and P. S. Simeonov, Theory of Impulsive Differential Equations, World Scientific, Publishing Company Limited, Singapore, 1989.

[4] B. O. Oyelami, "On military model for impulsive reinforcement functions using exclusion and marginalization techniques," Nonlinear Analysis: Theory, Methods and Applications, vol. 35, no. 8, pp. 947-958, 1999.

[5] B. O. Oyelami and S. O. Ale, "Solutions of impulsive diffusion and Von-Foerster-Makendrick models using the B-transform," Applied Mathematics, vol. 4, no. 12, pp. 1637-1646, 2013.
[6] B. O. Oyelami, "On singular impulsive systems using isomorphism decomposition method," Asian Journal of Mathematics and Computer Research, vol. 11, pp. 78-96, 2016.

[7] A. M. Samoilenko and N. A. Perestyuk, Impulsive Differential Equations, World Scientific Publishing Company Ltd, Singapore, 1995.

[8] I. M. Esuabana and J. A. Ugboh, "Survey of impulsive differential equations with continuous delay," International Journal of Mathematics Trends and Technology, vol. 60, no. 1, pp. 22-28, 2018.

[9] A. E. Coddington and N. Levinson, Theory of Ordinary Differential Equations, McGraw-Hill Book Company, New York, Ny, USA, 1955.

[10] Z. Lipcsey, I. M. Esuabana, J. A. Ugboh, and I. O. Isaac, "Integral representation of functions of bounded variation," Hindawi, Journal of Mathematics, vol. 2019, Article ID 1065946, 11 pages, 2019.

[11] R. F. Bass, Real Analysis for Graduate Students: Measure and Integration Theory, Createspace Ind Pub, Scotts Valley, CA, USA, 2011.

[12] U. A. Abasiekwere, I. M. Esuabana, I. O. Isaac, and Z. Lipcsey, "Existence theorem for linear neutral impulsive differential equations of the second order," Communications in Applied Analysis, vol. 22, no. 2, 2018.

[13] A. S. Abdel-Rady, A. M. A. El-Sayed, S. Z. Rida, and I. Ameen, "On some impulsive differential equations," Mathematical Sciences Letters, vol. 1, no. 2, pp. 105-111, 2012.

[14] D. D. Bainov and I. M. Stamova, "Existence, uniqueness and continuability of solutions of impulsive differential-difference equations," Journal of Applied Mathematics and Stochastic Analysis, vol. 12, no. 3, pp. 293-300, 1999.

[15] I. M. Esuabana, U. A. Abasiekwere, J. A. Ugboh, and Z. Lipcsey, "Equivalent construction of ordinary differential equations from impulsive systems," Academic Journal of Mathematical Sciences, vol. 4, no. 8, pp. 77-89, 2018.

[16] B. Li, "Existence of solutions for impulsive fractional evolution equations with periodic boundary condition," Advances in Difference Equations, vol. 2017, no. 236, Article ID 236, 2017.

[17] M. J. Mardanov, N. I. Mahmudov, and Y. A. Sharifov, "Existence and uniqueness theorems for impulsive fractional differential equations with the two-point and integral boundary conditions," The Scientific World Journal, vol. 2014, Article ID 918730, 8 pages, 2014.

[18] M. J. Mardonov, Y. A. Sharifov, and K. E. Ismayliov, "Existence and uniqueness of solutions for non-linear impulsive differential equations with three-point boundary conditions," E-Journal of Analysis and Mathematics, vol. 1, pp. 21-28, 2018.

[19] D. Zhang and B. Dai, "Existence of solutions for nonlinear impulsive differential equations with dirichlet boundary conditions," Mathematical and Computer Modelling, vol. 53, no. 5-6, pp. 1154-1161, 2011.

[20] A. Anokhin, L. Berezansky, and E. Braverman, "Stability of linear delay impulsive differential equations," Dynamical Systems and Applications, vol. 4, pp. 173-187, 1995.

[21] A. Anokhin, L. Berezansky, and E. Braverman, "Exponential stability of linear delay impulsive differential equations," Journal of Mathematical Analysis and Applications, vol. 193, no. 3, pp. 923-941, 1995.

[22] C. T. H. Baker, C. A. H. Paul, and D. R. Wille, "Issues in the numerical solution of evolutionary delay differential equations," Advances in Computational Mathematics, vol. 3, no. 3, pp. 171-196, 1995. 
[23] H. Willé and F. Karakoc, "Asymptotic constancy for impulsive delay differential equations," Dynamic Systems and Applications, vol. 17, pp. 71-84, 2008.

[24] L. Berezansky and E. Braverman, "Impulsive stabilization of linear delay differential equations," Dynamic Systems Applications, vol. 5, pp. 263-276, 1996.

[25] L. Berezansky and E. Braverman, "Exponential boundedness of solutions for impulsive delay differential equations," Applied Mathematics Letters, vol. 9, no. 6, pp. 91-95, 1996.

[26] B. Du and X. Zhang, Delay Dependent Stability Analysis and Synthesis for Uncertain Impulsive Switched System with Mixed Delays, Hindawi Publishing Corporation-Discrete Dynamic in Nature and Society, London, UK, 2011.

[27] F. Dubeau and J. Karrakchou, "State-dependent impulsive delay-differential equations," Applied Mathematics Letters, vol. 15, no. 3, pp. 333-338, 2002.

[28] J. R. Graef, M. K. Grammatikopoulos, and P. W. Spikes, "Asymptotic properties of solutions of nonlinear neutral delay differential equations of the second order," Radovi Matematicki, vol. 4, pp. 133-149, 1988.

[29] M. K. Grammatikopoulos, G. Ladas, and A. Meimaridou, "Oscillations of second order neutral delay differential equations," Radovi Matematicki, vol. 1, pp. 267-274, 1985.

[30] I. O. Isaac and Z. Lipcsey, "Linearized oscillations in nonlinear neutral delay impulsive differential equations," Journal of Modern Mathematics and Statistics-Medwell Journals-Pakistan, vol. 3, no. 1, pp. 1-7, 2009.

[31] I. O. Isaac and Z. Lipcsey, "Oscillations in linear neutral delay impulsive differential equations with constant coefficients," Communications in Applied Analysis, vol. 14, no. 2, pp. 123136, 2010.

[32] I. O. Isaac and Z. Lipcsey, "The existence of positive solutions to neutral delay impulsive differential equations," Communication in Applied Analysis, vol. 16, no. 1, pp. 23-46, 2012.

[33] I. O. Isaac, Z. Lipcsey, and U. Ibok, "Linearized oscillations in autonomous delay impulsive differential equations," British Journal of Mathematics \& Computer Science, vol. 4, no. 21, pp. 3068-3076, 2014.

[34] Q. Wang and X. Liu, "Impulsive stabilization of delay differential systems via the Lyapunov-Razumikhin method," Applied Mathematics Letters, vol. 20, no. 8, pp. 839-845, 2007.

[35] A. Weng and J. Sun, "Impulsive stabilization of second-order delay differential equations," Nonlinear Analysis: Real World Applications, vol. 8, no. 5, pp. 1410-1420, 2007.

[36] J. Yan, "Oscillation properties of a second-order impulsive delay differential equation," Computers \& Mathematics with Applications, vol. 47, no. 2-3, pp. 253-258, 2004.

[37] A. Zhao and J. Yan, "Asymptotic behavior of solutions of impulsive delay differential equations," Journal of Mathematical Analysis and Applications, vol. 201, no. 3, pp. 943-954, 1996.

[38] G. Ballinger and X. Liu, "Existence and uniqueness results for impulsive delay differential equations," DCDIS, vol. 5, pp. 579-591, 1999.

[39] B. S. Nagy, Introduction to Real Functions and Orthogonal Expansions, Oxford University Press, Oxford, UK, 1965.

[40] Z. Lipcsey, I. M. Esuabana, J. A. Ugboh, and I. O. Isaac, "Absolute continuous representation of functions of bounded variation," In press, 2019.

[41] J. C. Fournier, Graphs Theory and Applications, Wiley-ISTE, Hoboken, NJ, USA, 2013. 